EUROPEAN ORGANIZATION FOR NUCLEAR PHYSICS

CERN PPE/94-120

27 July 1994

\title{
Measurement of the Tau Lepton Polarization and its Forward-Backward Asymmetry from $Z^{0}$ Decays
}

\section{The OPAL Collaboration}

\begin{abstract}
We report on a measurement of the tau lepton polarization and its forward-backward asymmetry at the $Z^{0}$ resonance using the OPAL detector. This measurement is based on analyses of the $\tau \rightarrow \mathrm{e} \bar{\nu}_{e} \nu_{\tau}, \tau \rightarrow \mu \bar{\nu}_{\mu} \nu_{\tau}, \tau \rightarrow \pi(\mathrm{K}) \nu_{\tau}$ and $\tau \rightarrow \rho \nu_{\tau}$ decays from a sample of $30663 \mathrm{e}^{+} \mathrm{e}^{-} \rightarrow \tau^{+} \tau^{-}$events collected during the years 1990 to 1992. Assuming that the tau lepton decays according to $\mathrm{V}-\mathrm{A}$ theory, we measure the average $\tau$ polarization to be $\left\langle P_{\tau}\right\rangle=(-14.9 \pm 1.9 \pm 1.3) \%$ and the $\tau$ polarization forward-backward asymmetry to be $\mathrm{A}_{\text {pol }}^{F B}=(-8.9 \pm 2.2 \pm 0.9) \%$, where the first error is statistical and the second systematic. These results are consistent with the hypothesis of lepton universality. When combined under the assumption of universality, our results can be interpreted as a measurement of $\sin ^{2} \theta_{\text {eff }}^{\text {lept }}=0.2321 \pm 0.0023$ within the context of the Standard Model.
\end{abstract}

To be submitted to Z. Phys. C - Particles and Fields 


\section{The OPAL Collaboration}

R. Akers ${ }^{16}$, G. Alexander ${ }^{23}$, J. Allison ${ }^{16}$, K.J. Anderson ${ }^{9}$, S. Arcelli ${ }^{2}$, S. Asai ${ }^{24}$, A. Astbury ${ }^{28}$, D. Axen ${ }^{29}$, G. Azuelos ${ }^{18, a}$, A.H. Ball ${ }^{17}$, E. Barberio ${ }^{26}$, R.J. Barlow ${ }^{16}$, R. Bartoldus ${ }^{3}$, J.R. Batley ${ }^{5}$, G. Beaudoin ${ }^{18}$, A.Beck ${ }^{23}$, G.A. Beck ${ }^{13}$, J.Becker ${ }^{10}$, C.Beeston ${ }^{16}$, T. Behnke ${ }^{27}$, K.W. Bell ${ }^{20}$, G. Bella ${ }^{23}$,

P. Bentkowski ${ }^{18}$, S. Bentvelsen ${ }^{8}$, P. Berlich ${ }^{10}$, S. Bethke ${ }^{32}$, O. Biebel ${ }^{32}$, I.J. Bloodworth ${ }^{1}$, P. Bock ${ }^{11}$,

H.M. Bosch ${ }^{11}$, M. Boutemeur ${ }^{18}$, S. Braibant ${ }^{12}$, P. Bright-Thomas ${ }^{25}$, R.M. Brown ${ }^{20}$, A. Buijs ${ }^{8}$,

H.J. Burckhart ${ }^{8}$, C.Burgard ${ }^{27}$, P. Capiluppi ${ }^{2}$, R.K. Carnegie ${ }^{6}$, A.A. Carter ${ }^{13}$, J.R. Carter ${ }^{5}$,

C.Y.Chang ${ }^{17}$, C. Charlesworth ${ }^{6}$, D.G. Charlton ${ }^{8}$, S.L. Chu ${ }^{4}$, P.E.L.Clarke ${ }^{15}$, J.C.Clayton ${ }^{1}$, S.G. Clowes ${ }^{16}$, I. Cohen ${ }^{23}$, J.E. Conboy ${ }^{15}$, M. Coupland ${ }^{14}$, M.Cuffiani ${ }^{2}$, S. Dado ${ }^{22}$, C. Dallapiccola ${ }^{17}$, G.M. Dallavalle ${ }^{2}$, C. Darling ${ }^{31}$, S. De Jong ${ }^{13}$, H. Deng ${ }^{17}$, M. Dittmar ${ }^{4}$, M.S. Dixit ${ }^{7}$, E. do Couto e Silva $^{12}$, J.E. Duboscq ${ }^{8}$, E. Duchovni ${ }^{26}$, G. Duckeck ${ }^{8}$, I.P. Duerdoth ${ }^{16}$, U.C. Dunwoody ${ }^{5}$, P.A. Elcombe ${ }^{5}$,

P.G.Estabrooks ${ }^{6}$, E. Etzion ${ }^{23}$, H.G. Evans ${ }^{9}$, F.Fabbri ${ }^{2}$, B. Fabbro ${ }^{21}$, M. Fanti², M. Fierro ${ }^{2}$, M. Fincke-Keeler ${ }^{28}$, H.M. Fischer ${ }^{3}$, P. Fischer ${ }^{3}$, R. Folman ${ }^{26}$, D.G. Fong ${ }^{17}$, M. Foucher ${ }^{17}$, H. Fukui ${ }^{24}$, A. Fürtjes ${ }^{8}$, P. Gagnon ${ }^{6}$, A. Gaidot ${ }^{21}$, J.W. Gary ${ }^{4}$, J. Gascon ${ }^{18}$, N.I. Geddes ${ }^{20}$, C. Geich-Gimbel ${ }^{3}$, S.W. Gensler ${ }^{9}$, F.X. Gentit ${ }^{21}$, T. Geralis ${ }^{20}$, G. Giacomelli ${ }^{2}$, P. Giacomelli ${ }^{4}$, R. Giacomelli ${ }^{2}$, V. Gibson ${ }^{5}$,

W.R. Gibson ${ }^{13}$, J.D. Gillies ${ }^{20}$, J. Goldberg ${ }^{22}$, D.M. Gingrich ${ }^{30, a}$, M.J. Goodrick ${ }^{5}$, W. Gorn ${ }^{4}$, C. Grandi ${ }^{2}$, P. Grannis ${ }^{8}$, E. Gross ${ }^{26}$, J.Hagemann ${ }^{27}$, G.G.Hanson ${ }^{12}$, M. Hansroul ${ }^{8}$, C.K. Hargrove ${ }^{7}$,

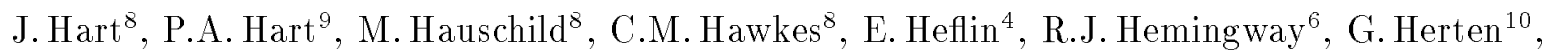
R.D. Heuer ${ }^{8}$, J.C.Hill ${ }^{5}$, S.J. Hillier ${ }^{8}$, T. Hilse ${ }^{10}$, D.A.Hinshaw ${ }^{18}$, P.R. Hobson ${ }^{25}$, D. Hochman ${ }^{26}$, A.Höcker ${ }^{3}$, R.J. Homer ${ }^{1}$, A.K.Honma ${ }^{28, a}$, R.E. Hughes-Jones ${ }^{16}$, R. Humbert ${ }^{10}$, P. Igo-Kemenes ${ }^{11}$, H. Thssen ${ }^{11}$, D.C.Imrie ${ }^{25}$, A. Jawahery ${ }^{17}$, P.W. Jeffreys ${ }^{20}$, H. Jeremie ${ }^{18}$, M. Jimack ${ }^{1}$, M. Jones ${ }^{6}$, R.W.L. Jones ${ }^{8}$, P. Jovanovic ${ }^{1}$, C. Jui ${ }^{4}$, D. Karlen ${ }^{6}$, K. Kawagoe ${ }^{24}$, T. Kawamoto ${ }^{24}$, R.K. Keeler ${ }^{28}$, R.G. Kellogg ${ }^{17}$, B.W. Kennedy ${ }^{20}$, B. King ${ }^{8}$, J. King ${ }^{13}$, S. Kluth ${ }^{5}$, T. Kobayashi ${ }^{24}$, M. Kobel ${ }^{10}$, D.S. Koetke ${ }^{8}$, T.P. Kokott ${ }^{3}$, S. Komamiya ${ }^{24}$, R. Kowalewski ${ }^{8}$, R. Howard ${ }^{29}$, P. Krieger ${ }^{6}$, J.von

Krogh $^{11}$, P. Kyberd ${ }^{13}$, G.D. Lafferty ${ }^{16}$, H. Lafoux ${ }^{8}$, R. Lahmann ${ }^{17}$, J. Lauber ${ }^{8}$, J.G. Layter ${ }^{4}$, P. Leblanc ${ }^{18}$, P. Le Du ${ }^{21}$, A.M. Lee ${ }^{31}$, E. Lefebvre ${ }^{18}$, M.H. Lehto ${ }^{15}$, D. Lellouch ${ }^{26}$, C. Leroy ${ }^{18}$, J. Letts ${ }^{4}$, L. Levinson ${ }^{26}$, Z. $\mathrm{Li}^{12}$, F. Liu $^{29}$, S.L. Lloyd ${ }^{13}$, F.K. Loebinger ${ }^{16}$, G.D.Long ${ }^{17}$, B. Lorazo ${ }^{18}$, M.J. Losty ${ }^{7}$,

X.C. Lou $^{8}$, J. Ludwig ${ }^{10}$, A. Luig ${ }^{10}$, M. Mannelli ${ }^{8}$, S. Marcellini ${ }^{2}$, C. Markus ${ }^{3}$, A.J. Martin ${ }^{13}$, J.P. Martinn ${ }^{18}$, T. Mashimo ${ }^{24}$, P. Mättig ${ }^{3}$, U. Maur ${ }^{3}$, J. McKenna ${ }^{29}$, T.J.McMahon ${ }^{1}$, A.I.McNab ${ }^{13}$, J.R. McNutt ${ }^{25}$, F. Meijers ${ }^{8}$, F.S. Merritt ${ }^{9}$, H. Mes $^{7}$, A.Michelini ${ }^{8}$, R.P. Middleton ${ }^{20}$, G. Mikenberg ${ }^{26}$,

J. Mildenberger ${ }^{6}$, D.J. Miller ${ }^{15}$, R. Mir ${ }^{26}$, W.Mohr ${ }^{10}$, C.Moisan ${ }^{18}$, A. Montanari' ${ }^{2}$, T. Mori ${ }^{24}$,

M. Moriï ${ }^{24}$, U. Müller ${ }^{3}$, B. Nellen ${ }^{3}$, B. Nijjhar ${ }^{16}$, S.W.O'Neale ${ }^{1}$, F.G.Oakham ${ }^{7}$, F. Odorici ${ }^{2}$, H.O. Ogren ${ }^{12}$, C.J. Oram ${ }^{28, a}$, M.J. Oreglia ${ }^{9}$, S. Orito ${ }^{24}$, J.P. Pansart ${ }^{21}$, G.N. Patrick ${ }^{20}$, M.J. Pearce ${ }^{1}$, P.Pfister ${ }^{10}$, P.D.Phillips ${ }^{16}$, J.E.Pilcher ${ }^{9}$, J.Pinfold ${ }^{30}$, D. Pitman ${ }^{28}$, D.E.Plane ${ }^{8}$, P. Poffenberger ${ }^{28}$, B. Poli ${ }^{2}$, A. Posthaus ${ }^{3}$, T.W.Pritchard ${ }^{13}$, H.Przysiezniak ${ }^{18}$, M.W. Redmond ${ }^{8}$, D.L. Rees ${ }^{8}$, D. Rigby ${ }^{1}$, M. Rison ${ }^{5}$, S.A. Robins ${ }^{13}$, D. Robinson ${ }^{5}$, J.M. Roney ${ }^{28}$, E. Ros ${ }^{8}$, S. Rossberg ${ }^{10}$, A.M. Rossi ${ }^{2}$, M.Rosvick ${ }^{28}$, P. Routenburg ${ }^{30}$, Y. Rozen ${ }^{8}$, K. Runge ${ }^{10}$, O.Runolfsson ${ }^{8}$, D.R. Rust ${ }^{12}$, M.Sasaki ${ }^{24}$, C.Sbarra ${ }^{2}$, A.D.Schaile ${ }^{8}$, O.Schaile ${ }^{10}$, F.Scharf ${ }^{3}$, P.Scharff-Hansen ${ }^{8}$, P. Schenk ${ }^{4}$, B.Schmitt ${ }^{3}$, H.von der Schmitt ${ }^{11}$, M.Schröder ${ }^{12}$, H.C.Schultz-Coulon ${ }^{10}$, P.Schütz ${ }^{3}$, M.Schulz ${ }^{8}$, C.Schwick ${ }^{27}$, J.Schwiening ${ }^{3}$, W.G. Scott ${ }^{20}$, M.Settles ${ }^{12}$, T.G.Shears ${ }^{5}$, B.C.Shen ${ }^{4}$, C.H.Shepherd-Themistocleous ${ }^{7}$,

P.Sherwood ${ }^{15}$, G.P.Siroli ${ }^{2}$, A.Skillman ${ }^{16}$, A.Skuja ${ }^{17}$, A.M.Smith ${ }^{8}$, T.J.Smith ${ }^{28}$, G.A.Snow ${ }^{17}$, R. Sobie ${ }^{28}$, R.W.Springer ${ }^{17}$, M.Sproston ${ }^{20}$, A.Stahl ${ }^{3}$, C.Stegmann ${ }^{10}$, K.Stephens ${ }^{16}$, J.Stenerer ${ }^{28}$, B.Stockhausen ${ }^{3}$, R.Ströhmer ${ }^{11}$, D.Strom ${ }^{19}$, P.Szymanski ${ }^{20}$, H. Takeda ${ }^{24}$, T. Takeshita ${ }^{24}$, S. Tarem ${ }^{26}$,

M. Tecchio ${ }^{9}$, P. Teixeira-Dias ${ }^{11}$, N. Tesch ${ }^{3}$, M.A.Thomson ${ }^{15}$, S. Towers ${ }^{6}$, T. Tsukamoto ${ }^{24}$,

M.F. Turner-Watson ${ }^{8}$, D. Van den plas ${ }^{18}$, R. Van Kooten $^{12}$, G. Vasseur ${ }^{21}$, M. Vincter ${ }^{28}$, A.Wagner ${ }^{27}$, D.L. Wagner ${ }^{9}$, C.P. Ward ${ }^{5}$, D.R. Ward ${ }^{5}$, J.J. Ward ${ }^{15}$, P.M. Watkins ${ }^{1}$, A.T. Watson ${ }^{1}$, N.K. Watson ${ }^{7}$, P. Weber ${ }^{6}$, P.S. Wells ${ }^{8}$, N. Wermes ${ }^{3}$, B. Wilkens ${ }^{10}$, G.W.Wilson ${ }^{4}$, J.A.Wilson ${ }^{1}$, V-H. Winterer ${ }^{10}$, 
T. Wlodek ${ }^{26}$, G. Wolf ${ }^{26}$, S. Wotton ${ }^{11}$, T.R. Wyatt ${ }^{16}$, A. Yeaman ${ }^{13}$, G. Yekutieli ${ }^{26}$, M. Yurko ${ }^{18}$, W. Zeuner ${ }^{8}$, G.T.Zorn ${ }^{17}$.

${ }^{1}$ School of Physics and Space Research, University of Birmingham, Birmingham B15 2TT, UK ${ }^{2}$ Dipartimento di Fisica dell' Università di Bologna and INFN, I-40126 Bologna, Italy

${ }^{3}$ Physikalisches Institut, Universität Bonn, D-53115 Bonn, Germany

${ }^{4}$ Department of Physics, University of California, Riverside CA 92521, USA

${ }^{5}$ Cavendish Laboratory, Cambridge CB3 0HE, UK

${ }^{6}$ Carleton University, Department of Physics, Colonel By Drive, Ottawa, Ontario K1S 5B6, Canada

${ }^{7}$ Centre for Research in Particle Physics, Carleton University, Ottawa, Ontario K1S 5B6, Canada

${ }^{8}$ CERN, European Organisation for Particle Physics, CH-1211 Geneva 23, Switzerland

${ }^{9}$ Enrico Fermi Institute and Department of Physics, University of Chicago, Chicago IL 60637, USA

${ }^{10}$ Fakultät für Physik, Albert Ludwigs Universität, D-79104 Freiburg, Germany

${ }^{11}$ Physikalisches Institut, Universität Heidelberg, D-69120 Heidelberg, Germany

${ }^{12}$ Indiana University, Department of Physics, Swain Hall West 117, Bloomington IN 47405, USA

${ }^{13}$ Queen Mary and Westfield College, University of London, London E1 4NS, UK

${ }^{14}$ Birkbeck College, London WC1E 7HV, UK

${ }^{15}$ University College London, London WC1E 6BT, UK

${ }^{16}$ Department of Physics, Schuster Laboratory, The University, Manchester M13 9PL, UK

${ }^{17}$ Department of Physics, University of Maryland, College Park, MD 20742, USA

${ }^{18}$ Laboratoire de Physique Nucléaire, Université de Montréal, Montréal, Quebec H3C 3J7, Canada

${ }^{19}$ University of Oregon, Department of Physics, Eugene OR 97403, USA

${ }^{20}$ Rutherford Appleton Laboratory, Chilton, Didcot, Oxfordshire OX11 0QX, UK

${ }^{21}$ CEA, DAPNIA/SPP, CE-Saclay, F-91191 Gif-sur-Yvette, France

${ }^{22}$ Department of Physics, Technion-Israel Institute of Technology, Haifa 32000, Israel

${ }^{23}$ Department of Physics and Astronomy, Tel Aviv University, Tel Aviv 69978, Israel

${ }^{24}$ International Centre for Elementary Particle Physics and Department of Physics, University of Tokyo, Tokyo 113, and Kobe University, Kobe 657, Japan

${ }^{25}$ Brunel University, Uxbridge, Middlesex UB8 3PH, UK

${ }^{26}$ Particle Physics Department, Weizmann Institute of Science, Rehovot 76100, Israel

${ }^{27}$ Universität Hamburg/DESY, II Institut für Experimental Physik, Notkestrasse 85, D-22607 Hamburg, Germany

${ }^{28}$ University of Victoria, Department of Physics, P O Box 3055, Victoria BC V8W 3P6, Canada

${ }^{29}$ University of British Columbia, Department of Physics, Vancouver BC V6T 1Z1, Canada

${ }^{30}$ University of Alberta, Department of Physics, Edmonton AB T6G 2J1, Canada

${ }^{31}$ Duke University, Dept of Physics, Durham, NC 27708-0305, USA

${ }^{32}$ Technische Hochschule Aachen, III Physikalisches Institut, Sommerfeldstrasse 26-28, D-52056 Aachen, Germany

${ }^{a}$ Also at TRIUMF, Vancouver, Canada V6T 2 A3 


\section{Introduction}

One of the phenomena characterizing weak interactions is the non-conservation of parity. This effect, originally established for weak charged-current interactions, is also predicted in the Standard Model [1] to exist in neutral-current interactions, resulting in different $Z^{0}$ couplings to left-handed and right-handed fermions. Consequently, fermions produced in $Z^{0}$ decay are expected to have a degree of polarization which depends on their coupling constants. Similarly, $\mathrm{Z}^{0}$ particles produced by $\mathrm{e}^{+} \mathrm{e}^{-}$annihilations are expected to be polarized because of their different couplings to the incoming left-handed and right-handed electrons. One of the consequences of this $Z^{0}$ polarization is a forward-backward asymmetry in the polarization of the outgoing fermions. These polarization phenomena can be studied in the process $\mathrm{e}^{+} \mathrm{e}^{-} \rightarrow \tau^{+} \tau^{-}$using the energy distribution of the $\tau$ decay products in the laboratory frame and can be used to test electron-tau universality. Within the context of the Standard Model, they can also be used to extract a value for the effective electroweak mixing angle, $\sin ^{2} \theta_{\text {eff }}^{\text {lept }}$ [2].

The present paper describes a measurement of the $\tau$ polarization, $\left\langle P_{\tau}\right\rangle$, and its forwardbackward asymmetry, $A_{\text {pol }}^{F B}$, using the data collected with the OPAL detector at LEP during the period 1990-1992, based on a sample of $30663 \mathrm{e}^{+} \mathrm{e}^{-} \rightarrow \tau^{+} \tau^{-}$events which were detected within the polar angle range of $|\cos \theta|<0.68^{1}$. Most of these events $(93 \%)$ were measured on the $\mathrm{Z}^{0}$ peak and the remainder at centre-of-mass energies $\left(E_{c m}\right)$ within $3 \mathrm{GeV}$ above and below the peak of the $Z^{0}$ resonance. The decay channels $\tau \rightarrow \mathrm{e} \bar{\nu}_{e} \nu_{\tau}, \tau \rightarrow \mu \bar{\nu}_{\mu} \nu_{\tau}, \tau \rightarrow \pi(\mathrm{K}) \nu_{\tau}$ and $\tau \rightarrow \rho \nu_{\tau}$ are used. These new results supersede our first measurement [3] which was based solely on 1990 data and did not include the $\tau \rightarrow \rho \nu_{\tau}$ decay channel.

The analysis of $\tau \rightarrow \mathrm{e} \bar{\nu}_{e} \nu_{\tau}, \tau \rightarrow \mu \bar{\nu}_{\mu} \nu_{\tau}$ and $\tau \rightarrow \pi(\mathrm{K}) \nu_{\tau}$ decays is based on an event-by-event maximum likelihood fit to the theoretical energy distributions, corrected for radiative effects and detector response, in which the correlations between the two $\tau$ decays are taken into account. We present for the first time the details of this 'global fit' and apply it to the three channels from which the polarization is extracted using distributions in simple observables. The analysis includes the $\tau$ - $\tau$ spin correlation effects in those events where both $\tau$ decay modes have been identified. If these correlations are not taken into account, our statistical errors are underestimated by between five and ten percent. This method has the advantage that $\tau$-pair selection and identification criteria that introduce correlations between the polarization observables of the two $\tau$ leptons in the same event are explicitly taken into account. This is particularly important for the leptonic channels where requirements are made on the whole event in order to suppress backgrounds from electron-pair and $\mu$-pair events.

The extraction of $\left\langle P_{\tau}\right\rangle$ and $\mathrm{A}_{\text {pol }}^{F B}$ in the $\tau \rightarrow \rho \nu_{\tau}$ decay channel is performed separately in a fit to measurements of $\left\langle P_{\tau}\right\rangle_{\theta}$ as a function of the $\tau^{-}$scattering angle. The polarization in each scattering angle bin is obtained from the two-dimensional distribution in decay angles of the $\rho$ in the $\tau$ rest frame and the charged pion in the $\rho$ rest frame. In the future, we anticipate including the analysis of the $\tau \rightarrow \rho \nu_{\tau}$ channel in the global fit. For the analyses presented here, the $\tau \rightarrow \rho \nu_{\tau}$ channel yields polarization errors comparable in magnitude to those obtained from the global maximum likelihood analysis of the $\tau \rightarrow \mathrm{e} \bar{\nu}_{e} \nu_{\tau}, \tau \rightarrow \mu \bar{\nu}_{\mu} \nu_{\tau}$ and $\tau \rightarrow \pi(\mathrm{K}) \nu_{\tau}$ decays.

A discussion of the polarization formalism, which includes the definition of the various observables used in this analysis and the relations between them, is presented in the next section. Section 3 describes the OPAL detector and the specifics of the Monte Carlo simulation. The selection of the sample of $\tau$-pairs and the decay-mode identification criteria are discussed

\footnotetext{
${ }^{1}$ The coordinate system is defined with $z$ along the $\mathrm{e}^{-}$beam direction, $\theta$ and $\phi$ being the polar and azimuthal angles, respectively.
} 
in Section 4. The details of the $\left\langle P_{\tau}\right\rangle$ and $\mathrm{A}_{\text {pol }}^{F B}$ extraction from the $\tau \rightarrow \mathrm{e} \bar{\nu}_{e} \nu_{\tau}, \tau \rightarrow \mu \bar{\nu}_{\mu} \nu_{\tau}$ and $\tau \rightarrow \pi(\mathrm{K}) \nu_{\tau}$ data is discussed in Section 5 and from the $\tau \rightarrow \rho \nu_{\tau}$ data in Section 6 . The results are combined and interpreted in terms of the Standard Model in Section 7.

\section{Tau polarization formalism}

The cross-sections for the process $\mathrm{e}^{+} \mathrm{e}^{-} \rightarrow \tau^{+} \tau^{-}$where the $\tau^{-}$has a definite (positive or negative) helicity are denoted by $\sigma_{+}$and $\sigma_{-}$, respectively. The average $\tau^{-}$polarization is then defined by,

$$
\left\langle P_{\tau^{-}}\right\rangle=\frac{\sigma_{+}-\sigma_{-}}{\sigma_{t o t}},
$$

where $\sigma_{t o t}=\sigma_{+}+\sigma_{-}$. Using this definition, $\sigma_{+}$and $\sigma_{-}$can be expressed as

$$
\begin{aligned}
& \sigma_{+}=\frac{1}{2}\left(1+\left\langle P_{\tau^{-}}\right\rangle\right) \sigma_{t o t} \\
& \text { and } \\
& \sigma_{-}=\frac{1}{2}\left(1-\left\langle P_{\tau^{-}}\right\rangle\right) \sigma_{t o t} .
\end{aligned}
$$

One has similar expressions involving the average $\tau^{+}$polarization. However, since the $\tau$ pairs are produced through an intermediate state of a spin-one boson (a photon or $\mathrm{Z}^{0}$ ), helicity conservation at high energies restricts the helicities of the $\tau^{+}$and the $\tau^{-}$in each $\tau$-pair to be almost always opposite. Consequently,

$$
\left\langle P_{\tau}\right\rangle \equiv\left\langle P_{\tau^{-}}\right\rangle=-\left\langle P_{\tau^{+}}\right\rangle
$$

This correlation is taken into account in our analysis, as will be described below.

The spin-one nature of the intermediate state also implies the following $\cos \theta$ distribution,

$$
\frac{1}{\sigma_{t o t}} \frac{d \sigma}{d \cos \theta}=\frac{3}{8}\left(1+\cos ^{2} \theta+\frac{8}{3} A_{F B} \cos \theta\right)
$$

where $\theta$ is the angle between the incoming electron and the outgoing $\tau^{-}$. The symbol $A_{F B} \equiv$ $\left(\sigma^{F}-\sigma^{B}\right) / \sigma_{\text {tot }}$ represents the forward-backward asymmetry, with $\sigma_{F}$ and $\sigma_{B}$ representing the cross-sections for forward $(\cos \theta>0)$ and backward $(\cos \theta<0)$ scattering events, respectively. Similar relations also hold for $\sigma_{+}$and $\sigma_{-}$:

$$
\begin{aligned}
& \frac{1}{\sigma_{t o t}} \frac{d \sigma_{+}}{d \cos \theta}=\frac{3}{16}\left[\left(1+\left\langle P_{\tau}\right\rangle\right)\left(1+\cos ^{2} \theta\right)+\frac{8}{3}\left(A_{F B}+A_{p o l}^{F B}\right) \cos \theta\right] \\
& \frac{1}{\sigma_{t o t}} \frac{d \sigma_{-}}{d \cos \theta}=\frac{3}{16}\left[\left(1-\left\langle P_{\tau}\right\rangle\right)\left(1+\cos ^{2} \theta\right)+\frac{8}{3}\left(A_{F B}-A_{p o l}^{F B}\right) \cos \theta\right]
\end{aligned}
$$

where

$$
A_{\text {pol }}^{F B} \equiv \frac{\left(\sigma_{+}^{F}-\sigma_{-}^{F}\right)-\left(\sigma_{+}^{B}-\sigma_{-}^{B}\right)}{\sigma_{t o t}},
$$

represents the forward-backward polarization asymmetry. From these expressions the average polarization for a given polar angle $\theta$ is given by,

$$
\left\langle P_{\tau}\right\rangle_{\theta}=\frac{\left\langle P_{\tau}\right\rangle\left(1+\cos ^{2} \theta\right)+\frac{8}{3} \mathrm{~A}_{p o l}^{F B} \cos \theta}{\left(1+\cos ^{2} \theta\right)+\frac{8}{3} \mathrm{~A}_{F B} \cos \theta} .
$$


The Standard Model gives predictions for $\left\langle P_{\tau}\right\rangle$ and $\mathrm{A}_{\text {pol }}^{F B}$ as functions of $s=E_{\mathrm{cm}}^{2}$ in terms of the mass and width of the $Z^{0}$ and its vector $\left(g_{v}\right)$ and axial-vector $\left(g_{a}\right)$ couplings to the electron and the tau lepton. For $s=m_{Z}^{2}$, in the improved Born approximation [4] (which accounts for the most significant weak radiative corrections) and neglecting the contributions of the intermediate photon, photon- $Z^{0}$ interference and other photonic radiative corrections, the Standard Model predicts:

$$
\left\langle P_{\tau}\right\rangle=-\mathcal{A}_{\tau} \quad \mathrm{A}_{\text {pol }}^{F B}=-\frac{3}{4} \mathcal{A}_{e}
$$

where,

$$
\mathcal{A}_{\ell} \equiv \frac{2 \hat{\mathrm{g}}_{v}^{\ell} / \hat{\mathrm{g}}_{a}^{\ell}}{1+\left(\hat{\mathrm{g}}_{v}^{\ell} / \hat{\mathrm{g}}_{a}^{\ell}\right)^{2}}
$$

and the ratio between the effective vector and axial-vector couplings [4], $\hat{\mathrm{g}}_{v}^{\ell} / \hat{\mathrm{g}}_{a}^{\ell}$, is related to the effective electroweak mixing angle by,

$$
\hat{\mathrm{g}}_{v}^{\ell} / \hat{\mathrm{g}}_{a}^{\ell}=1-4 \sin ^{2} \theta_{\mathrm{eff}}^{\mathrm{lept}}
$$

Therefore, our measurements of $\left\langle P_{\tau}\right\rangle$ and $\mathrm{A}_{\text {pol }}^{F B}$ provide a test of $\mathrm{e}-\tau$ universality in the neutral current which is independent of lepton universality tests we have performed by studying the line shapes and the forward-backward asymmetries of the electron-pair, $\mu$-pair and $\tau$-pair cross-sections [5].

For the measurements of $\left\langle P_{\tau}\right\rangle$ and $A_{\text {pol }}^{F B}$ the distributions described by equations 5 and 6 cannot be directly measured as it is not possible to determine the $\tau$ helicity on an event-byevent basis. Instead, distributions of kinematic variables of the $\tau$ decay products which depend on the $\tau$ helicity are used. These variables, as well as their distributions, depend on the decay mode used ${ }^{2}$. The $\tau \rightarrow \pi(\mathrm{K}) \nu_{\tau}$ and $\tau \rightarrow \rho \nu_{\tau}$ decays are the most sensitive modes for determining the $\tau$ polarization and, of the modes considered here, the purely leptonic decays have the lowest sensitivities because they contain two undetected neutrinos $[6,7]$.

For leptonic decays, $\tau \rightarrow \ell \bar{\nu}_{\ell} \nu_{\tau}(\ell=\mathrm{e}, \mu)$, the relevant kinematic variable is the lepton energy, scaled by the beam energy, $x_{\ell}=E_{\ell} / E_{\text {beam }}$. Assuming an exact $V-A$ structure of the charged current in $\tau$ decay, the distribution in $x_{\ell}$ is [6]

$$
\frac{1}{\Gamma_{\ell}} \frac{d \Gamma_{\ell}}{d x_{\ell}}=\frac{1}{3}\left(5-9 x_{\ell}^{2}+4 x_{\ell}^{3}\right)+\mathrm{P}_{\tau} \frac{1}{3}\left(1-9 x_{\ell}^{2}+8 x_{\ell}^{3}\right) \quad\left(0 \leq x_{\ell} \leq 1\right)
$$

where radiative effects and terms of order $m_{\ell} / m_{\tau}$ have been neglected but are, however, taken into account in the analysis.

An analogous kinematic variable, $E_{h} / E_{\text {beam }}$, can be used for semi-leptonic decays, $\tau \rightarrow \mathrm{h} \nu_{\tau}$, where $h$ represents a charged hadron. Here $E_{h} / E_{\text {beam }}$ is related to $\cos \theta^{*}$, where $\theta^{*}$ is the decay angle of the hadron in the $\tau$ rest frame,

$$
\cos \theta^{*}=\frac{2 E_{h} / E_{\text {beam }}-1-m_{h}^{2} / m_{\tau}^{2}}{\beta\left(1-m_{h}^{2} / m_{\tau}^{2}\right)}
$$

where $\beta$ is the velocity of the $\tau$. Assuming that the charged current in $\tau$ decay has an exact $\mathrm{V}-\mathrm{A}$ structure, the $\cos \theta^{*}$ distribution is $[6]$

$$
\frac{1}{\Gamma_{h}} \frac{d \Gamma_{h}}{d \cos \theta^{*}}=\frac{1}{2}\left(1+\mathrm{P}_{\tau} \alpha \cos \theta^{*}\right) \quad\left(-1 \leq \cos \theta^{*} \leq 1\right),
$$

\footnotetext{
${ }^{2}$ Note that the distributions are the same for the $\tau^{+}$and $\tau^{-}$provided that $\left\langle P_{\tau}\right\rangle$ is taken as the $\tau^{-}$helicity.
} 
where the factor $\alpha$ depends on the spin and mass of the charged hadron.

For $\tau \rightarrow \pi(\mathrm{K}) \nu_{\tau}$ decays $\alpha=1$. As there is no distinction made between the pion and kaon in this analysis, $m_{h}$ is not known on an event-by-event basis and the $\pi / K$ mixture is taken into account using the distribution,

$$
\frac{1}{\Gamma_{h}} \frac{d \Gamma_{h}}{d x_{h}}=\left(1-b_{K}\right) \frac{1}{\Gamma_{\pi}} \frac{d \Gamma_{\pi}}{d x_{h}}+b_{K} \frac{1}{\Gamma_{K}} \frac{d \Gamma_{K}}{d x_{h}}
$$

where $x_{h}$ represents the hadron momentum scaled by the beam energy and

$$
b_{K} \equiv \mathrm{BR}(\tau \rightarrow K \nu) /[\mathrm{BR}(\tau \rightarrow \pi \nu)+\mathrm{BR}(\tau \rightarrow K \nu)]=0.053 \pm 0.018[8] .
$$

For $\tau \rightarrow \rho \nu_{\tau}$ decays a value of $\cos \theta^{*}$ is calculated using the measured energy and invariant mass of the $\pi^{ \pm} \pi^{0}$ decay products of the $\rho$. A consequence of the $\rho$ mass and its being a vector particle is that the $\alpha$ factor in equation 14 is approximately equal to 0.46 [9]. This can be understood by recognizing that for the longitudinal $\rho$ polarization $\left(P_{\rho}=0\right)$, the $\cos \theta^{*}$ distribution is the same as for $\pi(\mathrm{K})$ (equation 14), whereas for transverse polarization $\left(P_{\rho}=\right.$ $\pm 1)$, the $\cos \theta^{*}$-term has an opposite sign. Therefore, with a mixture of the two $\rho$ polarizations, the dependence on $\cos \theta^{*}$ is reduced, losing sensitivity to $\mathrm{P}_{\tau}$. One way to recuperate most of the sensitivity is to use a second kinematic variable which is sensitive to the $\rho$ polarization. Following reference [7], the angle $\psi$ between the charged pion in the $\rho$ rest frame and the $\rho$ direction as measured in the laboratory frame is used.

For each $\tau$ decay channel, $i$, the distribution of the kinematic variable, $x_{i}$, is linear in $\mathrm{P}_{\tau}$, and can be written in the form,

$$
\frac{1}{\Gamma_{i}} \frac{d \Gamma_{i}}{d x_{i}}=f_{i}\left(x_{i}\right)+\mathrm{P}_{\tau} g_{i}\left(x_{i}\right)
$$

In general, $f_{i}\left(x_{i}\right)$ and $g_{i}\left(x_{i}\right)$ are different for each decay channel but for all decay modes the following normalization conditions are satisfied,

$$
\int f_{i}\left(x_{i}\right) d x_{i}=1 \quad \int g_{i}\left(x_{i}\right) d x_{i}=0 .
$$

The joint distributions of the $\tau$-pair production and decay is obtained from equations 5 and 6 by multiplying them with the corresponding decay distributions of the $\tau^{-}$and the $\tau^{+}$and summing up, resulting in,

$\frac{d^{3} \sigma_{i j}}{d \cos \theta d x_{i} d x_{j}}=\frac{3}{16} \sigma_{i j} \sum_{h e l= \pm}\left[\left(1 \pm\left\langle P_{\tau}\right\rangle\right)\left(1+\cos ^{2} \theta\right)+\frac{8}{3}\left(A_{F B} \pm A_{p o l}^{F B}\right) \cos \theta\right]\left[f_{i}\left(x_{i}\right) \pm g_{i}\left(x_{i}\right)\right]\left[f_{j}\left(x_{j}\right) \pm g_{j}\left(x_{j}\right)\right]$.

Here, $\sigma_{i j}$ is the cross-section to produce an $\mathrm{e}^{+} \mathrm{e}^{-} \rightarrow \tau^{+} \tau^{-}$event in which one $\tau$ decays via channel $i$ and the other via channel $j$. This expression includes the correlation between the decay distributions of the two $\tau$ leptons, which must be taken into account when analyzing events in which both $\tau$ decay channels are identified.

\section{The OPAL detector and Monte Carlo simulation}

The OPAL detector, which is described in detail in reference [10], is a solenoidal detector with a pressurized central tracking system operating in a $0.435 \mathrm{~T}$ magnetic field. A leadglass electromagnetic calorimeter (ECAL) together with presampler chambers and time-offlight scintillators (TOF) is located outside the magnet coil and pressure vessel. The magnet 
return yoke is instrumented for hadron calorimetry (HCAL) and is surrounded by external muon chambers. Calorimeters close to the beam axis measure luminosity and complete the acceptance. Full efficiency at the trigger level [11] is achieved for $\mathrm{e}^{+} \mathrm{e}^{-} \rightarrow \tau^{+} \tau^{-}$events within the angular acceptance of this analysis. Descriptions of those detector components which are used in the polarization analyses are presented in the rest of this section. The data sample is restricted to the periods when these components were fully operational.

The central tracking system is designed to measure the trajectory and specific energy loss of charged particles. It consists of a silicon micro-vertex detector, a precision vertex drift chamber, a large volume drift chamber (jet chamber) and $z$-chambers which measure the $z$ coordinate of charged particles as they leave the jet chamber. The jet chamber is $4 \mathrm{~m}$ long and $3.7 \mathrm{~m}$ in diameter and is divided into 24 azimuthal sectors. In the range $|\cos \theta|<0.73,159$ points are measured in the jet chamber along each track and at least 20 points on a track are obtained over $96 \%$ of the full solid angle. The momentum in the $r$ - $\phi$ plane, $p_{t}$, is measured with a resolution of $\left(\sigma\left(p_{t}\right) / p_{t}\right)^{2} \approx 0.02^{2}+\left(0.0015 \cdot p_{t}\right)^{2},\left(p_{t}\right.$ in $\left.\mathrm{GeV}\right)$ for $|\cos \theta|<0.73$.

The electromagnetic calorimeter, consisting of a barrel and two endcap arrays of lead glass blocks, covers the full azimuthal angular range in the polar angle range of $|\cos \theta|<0.82$ for the barrel and $0.81<|\cos \theta|<0.984$ for the endcaps. Each block subtends a solid angle of approximately $40 \times 40 \mathrm{mrad}^{2}$, with a thickness of over 24 radiation lengths in the barrel region and typically 22 radiation lengths in the endcaps. The barrel lead-glass blocks have a pointing geometry, but in order to achieve good hermeticity, the small $1 \mathrm{~mm}$ gaps between the leadglass blocks do not point exactly to the interaction point. The intrinsic energy resolution of the calorimeter is $5-6 \% / \sqrt{E}$ ( $E$ in $\mathrm{GeV}$ ) which in the barrel region is degraded by about a factor of two by the $\approx 2 / \sin \theta$ radiation lengths of material in the magnet coil and the pressure vessel in front of the calorimeter. The angular resolution of electromagnetic clusters is approximately $4 \mathrm{mrad}$ both in $\theta$ and $\phi$ for energies above $10 \mathrm{GeV}$. The barrel presampler detector is a cylinder of limited streamer tubes located between the TOF and the barrel lead-glass calorimeter. It is used to obtain a precise angular measurement of electromagnetic showers originating in the coil with an angular resolution for high energy photons of about $2 \mathrm{mrad}$. For the measurement of electron energies in the $\tau \rightarrow \mathrm{e} \bar{\nu}_{e} \nu_{\tau}$ channel, the energy deposited in the ECAL is corrected using the presampler determination of energy loss in the pressure vessel and magnet coil.

The hadron calorimeter, which consists of nine layers (eight layers in the endcap) of streamer tubes interleaved with the iron slabs of the magnet return yoke, is read out via $4 \mathrm{~mm}$ wide strips and $50 \times 50 \mathrm{~cm}^{2}$ pads arranged in projective towers. These strips and pads provide measurements in the $(r, \phi)$ plane and the $z$ direction. The material in front of the hadron calorimeter corresponds to about two hadronic interaction lengths. In addition to the energy measurement, the strip readout permits the identification of minimum ionizing particles traversing the iron and thus helps with muon identification.

The muon chamber system in the barrel region consists of 220 planar drift chambers, each $60 \mathrm{~cm}$ wide with a single central wire. They are arranged in four layers over a radial distance of about $50 \mathrm{~cm}$. Most of the chambers are $10.4 \mathrm{~m}$ long, covering a range in polar angle of $|\cos \theta|<0.70$. In the space below the detector, where room is taken up by the magnet supports, the chambers are $6 \mathrm{~m}$ long and the detector is sensitive only for $|\cos \theta|<0.47$.

In order to simulate the various processes which potentially contribute to the selected $\tau$-pair data sample, several Monte Carlo data sets were used. The response of the OPAL detector to the generated particles in each case was modelled using a simulation program [12] based on the GEANT [13] package. In all cases, the Monte Carlo and real data were reconstructed and analysed in an identical manner. 
Initial estimates of the efficiencies and purities of the selections for the individual $\tau$ decay channels were obtained using the KORALZ 3.8 Monte Carlo generator $^{3}$ [14]. Tau polarization and its effect on the decay spectra, as well as the correlation between the two $\tau$ 's are included. The $\tau$-pair Monte Carlo sample generated for these studies is approximately seven times larger than the data sample.

In order to estimate the background coming from the multihadronic decays of the $Z^{0}$, the JETSET Monte Carlo [15] was used with the parameters tuned to fit the global event shape distributions of OPAL multihadron data [16]. Backgrounds from radiative $\mu$-pair and electron-pair events were estimated using events generated with the KORALZ 3.8 Monte Carlo program [14] and the BABAMC Monte Carlo program [17], respectively. Non-resonant t-channel two-photon processes were simulated with the generator described in reference [18].

\section{Tau-pair selection and decay mode identification}

In this analysis we initially select a sample of $\tau$-pair candidates from which samples of $\tau \rightarrow \mathrm{e} \bar{\nu}_{e} \nu_{\tau}$, $\tau \rightarrow \mu \bar{\nu}_{\mu} \nu_{\tau}, \tau \rightarrow \pi(\mathrm{K}) \nu_{\tau}$ and $\tau \rightarrow \rho \nu_{\tau}$ candidates are subsequently identified. The selection requirements are detailed in reference [3]. The general strategy is first to identify events characterized by a pair of back-to-back, narrow jets with low particle multiplicity ( $\tau$ jets $). A|\cos \theta|$ is calculated for each $\tau$ jet and the average of the two jets, $\overline{|\cos \theta|}$, must be less than 0.68 . The same $\overline{|\cos \theta|}$ is used as the estimator for the magnitude of the cosine of the $\tau^{-}$scattering angle in the analysis. Background from two-photon processes is then suppressed by requiring that the events have a minimum total energy and significant missing transverse momentum when the total energy in the event is low. After removing cosmic ray backgrounds, the events which remain are almost entirely lepton-pairs. The $\tau$-pair events are isolated by identifying and removing the $\mu$-pair and electron-pair events using their high energy characteristics. We select $30663 \tau$-pair events by these criteria. The $\mathrm{e}^{+} \mathrm{e}^{-} \rightarrow \tau^{+} \tau^{-}$selection is estimated from Monte Carlo studies $[14,12,13]$ to have a $54 \%$ efficiency (93\% within the geometrical acceptance) and a background level of $1.7 \%$. Details of the background in each decay channel are presented below. Note that the criteria designed to remove two-photon, electron-pair and $\mu$-pair events introduce kinematic biases which must be understood for the measurement of the polarization. The treatment of these criteria and the systematic uncertainties associated with them are considered in Section 5.

The $\tau \rightarrow \mathrm{e} \bar{\nu}_{e} \nu_{\tau}$ identification algorithm selects jets containing a track which deposits almost all of its energy within a highly localized region of the ECAL. The $\tau \rightarrow \mu \bar{\nu}_{\mu} \nu_{\tau}$ selection requires an isolated charged track with energy deposition in the ECAL and HCAL consistent with the passage of a minimum-ionizing particle and signals in the muon chambers associated to the track. The signature for a $\tau \rightarrow \pi(\mathrm{K}) \nu_{\tau}$ decay is not as distinct as that for either a $\tau \rightarrow \mathrm{e} \bar{\nu}_{e} \nu_{\tau}$ or a $\tau \rightarrow \mu \bar{\nu}_{\mu} \nu_{\tau}$ decay because hadronic interactions can begin in the magnet coil, ECAL or HCAL. While electrons and muons can be efficiently removed from the $\tau \rightarrow \pi(\mathrm{K}) \nu_{\tau}$ sample, the semileptonic $\tau$ decays containing neutral pions are problematic because at $\mathrm{E}_{c m}=91 \mathrm{GeV}$ the boost of the $\tau$ causes significant overlap in the ECAL of the charged pion hadronic shower and the electromagnetic showers from the photons produced in the $\pi^{0}$ decay. We exploit the fact that electromagnetic interactions begin in the coil with a higher probability than hadronic interactions. The presampler thereby provides an effective veto against decays containing neutral pions. The specific requirements which exploit these characteristics of the three decay modes

\footnotetext{
${ }^{3}$ KORALZ 3.8 takes into account initial state bremsstrahlung up to $\mathcal{O}\left(\alpha^{2}\right)$ (with exclusive exponentiation), final state bremsstrahlung and electroweak corrections up to $\mathcal{O}(\alpha)$, and single bremsstrahlung (in the leading logarithmic approximation) in $\tau$ decay for the decay modes used in this analysis.
} 
are described in reference [3]. In addition, for this analysis, we have introduced fiducial requirements designed to remove from the acceptance those regions of the detector which are either not well modelled in the Monte Carlo or where backgrounds from non-tau sources enter the sample. The characteristic global efficiencies for the $\tau \rightarrow \mathrm{e} \bar{\nu}_{e} \nu_{\tau}, \tau \rightarrow \mu \bar{\nu}_{\mu} \nu_{\tau}$ and $\tau \rightarrow \pi(\mathrm{K}) \nu_{\tau}$ are $66 \%, 72 \%$ and $30 \%$, respectively and include the effect of a requirement which removes candidates having $x<0.05$. These numbers include the preselection efficiency within the fiducial region of the analysis and ignore decay or detection correlations. The backgrounds are $5.0 \%$, $2.5 \%$ and $7.2 \%$, respectively. Plots of the global efficiencies as a function of $x$ in each of these channels are shown in Figure 1. The $x$ dependence of the efficiency is an important systematic of the polarization measurement and its control is discussed in detail in the subsequent section.

The results from the $\tau \rightarrow \rho \nu_{\tau}$ decay are based on two samples of $\tau \rightarrow \rho \nu_{\tau}$ candidate events, referred to as samples I and II as defined in reference [19]. Each sample is independently selected and values for $\left\langle P_{\tau}\right\rangle$ and $A_{\text {pol }}^{F B}$ are determined separately. Both selections have an efficiency of approximately $50 \%$ and about one third of the combined number of candidates is common to both samples. Since the $\rho$ decays into a charged and a neutral pion, the signature of the $\tau \rightarrow \rho \nu_{\tau}$ decay is a single charged track accompanied by ECAL energy deposition consistent with the interaction of the two photons from the neutral pion decay. The details of how these characteristics are exploited to obtain the two $\tau \rightarrow \rho \nu_{\tau}$ samples are discussed in reference [19] although slight modifications have been made here in order to optimize the analysis for the polarization measurement. The efficiency for selecting sample I is $48 \%$ where the background is $21 \%$. For sample II the efficiency is $58 \%$ and the background $23 \%$. Plots of the global efficiencies as a function of $\cos \theta^{*}$ and $\cos \psi$ for $\tau \rightarrow \rho \nu_{\tau}$ decays for the two selections are presented in Figure 2. Note that in this channel the $\tau \rightarrow \mathrm{K}^{*} \nu_{\tau} \rightarrow \mathrm{K} \pi^{0} \nu_{\tau}$ decay mode is treated as background.

In Table 1 we list the number of $\tau$-pair events in the data, according to their $\tau$ decay mode identifications. The label ' 0 ' refers to the case where one of the $\tau$ decays is not identified. The events in which neither jet is identified ( $22 \%$ of the whole sample) are not used in the analysis. As is evident from this table, the identification efficiencies of $\tau$ jets are not independent. The requirements that remove two-photon, electron-pair and $\mu$-pair events introduce these correlations. Such correlations are, however, taken into account in the analysis.

The helicity correlation between the decay distributions of the two $\tau$ 's for events where both $\tau$ 's decay to e, $\mu$ or $\pi(\mathrm{K})$ is taken into account in equation 18 and is incorporated into the analysis. The correlation in events where both $\tau$ decays are identified and one of them is $\tau \rightarrow \rho \nu_{\tau}$ is not taken into account explicitly. In order to prevent "double-counting" of the polarization information in these events, only the $\tau$ decaying into the more sensitive decay mode is used. Hence, events of the type $\ell$ - $\rho$, i.e. one $\tau$ decays to a lepton and the other $\tau$ decays to a $\rho$-meson, are discarded from the maximum likelihood analysis, and are used only in the $\tau \rightarrow \rho \nu_{\tau}$ analysis. Similarly, events of the type $\pi$ - $\rho$ are used only in the maximum likelihood analysis where the $\tau \rightarrow \pi(\mathrm{K}) \nu_{\tau}$ decay is considered. For events of the type $\rho$ - $\rho$ we weight each identified $\tau \rightarrow \rho \nu_{\tau}$ decay by 0.5 .

\section{$5 \quad\left\langle P_{\tau}\right\rangle$ and $\mathrm{A}_{p o l}^{F B}$ from $\tau \rightarrow \mathrm{e} \bar{\nu}_{e} \nu_{\tau}, \tau \rightarrow \mu \bar{\nu}_{\mu} \nu_{\tau}$ and $\tau \rightarrow \pi(\mathrm{K}) \nu_{\tau}$ decays}

For the analysis of the $\tau \rightarrow \mathrm{e} \bar{\nu}_{e} \nu_{\tau}, \tau \rightarrow \mu \bar{\nu}_{\mu} \nu_{\tau}$ and $\tau \rightarrow \pi(\mathrm{K}) \nu_{\tau}$ decays, $\left\langle P_{\tau}\right\rangle$ and $\mathrm{A}_{\text {pol }}^{F B}$ are determined using an event-by-event maximum likelihood fit to the data of the theoretical distribution (equation 18) corrected for the effects of radiation, efficiency, resolution and background. In 


\begin{tabular}{|c|c|c|c|c|c|c|}
\hline$\tau_{1}$ decay & & & & & & \\
\hline$\downarrow$ & & & & & & \\
\hline 0 & 6833 & & & & & \\
\hline e & 3585 & 574 & & & & \\
\hline & $54 / 19$ & 46 & & & & \\
\hline & 5 & 12 & & & & \\
\hline$\mu$ & 3897 & 1288 & 487 & & & \\
\hline & $58 / 23$ & 58 & 46 & & & \\
\hline & 3 & 6 & 5 & & & \\
\hline$\pi(K)$ & 1145 & 346 & 385 & 55 & & \\
\hline & $26 / 9$ & 21 & 25 & 11 & & \\
\hline & 7 & 11 & 8 & 14 & & \\
\hline$\rho$ & 5879 & 1960 & 1985 & 623 & 1621 & \\
\hline & $51 / 19$ & 44 & 51 & 20 & 41 & \\
\hline & 27 & 31 & 29 & 32 & 48 & \\
\hline & 0 & e & $\mu$ & $\pi(K)$ & $\rho$ & $\leftarrow \tau_{2}$ decay \\
\hline
\end{tabular}

Table 1: Number of $\tau$-pair events classified according to the identified decay channel is presented as the first number in each box. The percentage efficiencies and percentage backgrounds are presented on the second and third line, respectively. The efficiencies include the contributions from the $\tau$-pair selection for those events within $|\cos \theta|<0.68$. The efficiencies in the first column (i.e. when only one $\tau$ is identified) are subdivided into two classes: the first is for the case where the opposite $\tau$ does not decay into one of the four channels; the second is where the opposite $\tau$ decays into one of the four channels but is not identified. The label ' 0 ' refers to the case where the $\tau$ decay was not identified. For the $\tau \rightarrow \rho \nu_{\tau}$ decays, the numbers represent the contributions from both selection algorithms.

this method, the following expression is minimized,

$$
W=-\ln \mathcal{L}=-\sum_{n=1}^{N} \ln \left\{\frac{1}{\sigma^{\prime}{ }_{i j}} \frac{d^{3} \sigma_{i j}^{\prime}}{d \cos \theta d x_{i} d x_{j}}\right\}_{n},
$$

where $\mathcal{L}$ is the likelihood function. The sum in equation 19 runs over all selected $\tau$-pair events, where at least one of the two $\tau$ decays has been identified as a $\tau \rightarrow \mathrm{e} \bar{\nu}_{e} \nu_{\tau}, \tau \rightarrow \mu \bar{\nu}_{\mu} \nu_{\tau}$ or $\tau \rightarrow \pi(\mathrm{K}) \nu_{\tau}$ candidate. The term in the logarithm is the corrected differential cross-section, normalized to one. It corresponds to the case where both $\tau$ decays are identified and is replaced by $\left(1 / \sigma^{\prime}{ }_{i}\right) d^{2} \sigma^{\prime}{ }_{i} / d \cos \theta d x_{i}$ when only one $\tau$ decay is identified.

When both $\tau$ decays are identified, the corrected cross-section can be written in the following form,

$$
\begin{aligned}
\frac{16}{3} \frac{1}{\sigma_{i j}^{\prime}} \frac{d^{3} \sigma_{i j}^{\prime}}{d \cos \theta d x_{i} d x_{j}} & =\sum_{h e l= \pm}\left\{\left[\left(1 \pm\left\langle P_{\tau}\right\rangle\right)\left(1+\cos ^{2} \theta\right)+\frac{8}{3}\left(A_{F B} \pm A_{p o l}^{F B}\right) \cos \theta\right]\right. \\
& \times \mathcal{E}_{i j}^{ \pm}\left(x_{i}, x_{j}, \cos \theta\right)\left[h_{i}^{ \pm}\left(x_{i}\right) \epsilon_{i}\left(x_{i}, \cos \theta\right)+\beta_{i}^{ \pm}\left(x_{i}, \cos \theta\right)\right] \\
& \left.\times\left[h_{j}^{ \pm}\left(x_{j}\right) \epsilon_{j}\left(x_{j}, \cos \theta\right)+\beta_{j}^{ \pm}\left(x_{j}, \cos \theta\right)\right]\right\} \\
& +\beta_{i j}^{\text {non }-\tau}\left(x_{i}, x_{j}, \cos \theta\right) .
\end{aligned}
$$


Here $\mathcal{E}_{i j}^{ \pm}\left(x_{i}, x_{j}, \cos \theta\right)$ is the efficiency for selecting a $\tau$-pair event in which one $\tau$ decays via channel $i$ and the other via $j ; \epsilon_{i}\left(x_{i}, \cos \theta\right)$ is the efficiency for identifying channel $i ; \beta_{i}^{ \pm}\left(x_{i}, \cos \theta\right)$ is the distribution of background events from other $\tau$ decay channels when applying the channel $i$ selection procedure; and $\beta_{i j}^{n o n-\tau}\left(x_{i}, x_{j}, \cos \theta\right)$ corresponds to background contributions from events which are not $\tau$-pairs. The function $h_{i}^{ \pm}\left(x_{i}\right)$ is the distribution of measured $x$ for channel $i$, after applying corrections for detector resolution and radiative effects to $f_{i}\left(x_{i}\right) \pm g_{i}\left(x_{i}\right)$.

If only one $\tau$ decay is identified, the corrected differential cross-section takes a simpler form,

$$
\begin{aligned}
\frac{16}{3} \frac{1}{\sigma_{i}^{\prime}} \frac{d^{2} \sigma_{i}^{\prime}}{d \cos \theta d x_{i}} & =\sum_{h e l= \pm}\left\{\left[\left(1 \pm\left\langle P_{\tau}\right\rangle\right)\left(1+\cos ^{2} \theta\right)+\frac{8}{3}\left(A_{F B} \pm A_{p o l}^{F B}\right) \cos \theta\right]\right. \\
& \left.\times \mathcal{E}_{i}^{ \pm}\left(x_{i}, \cos \theta\right)\left[h_{i}^{ \pm}\left(x_{i}\right) \epsilon_{i}\left(x_{i}, \cos \theta\right)+\beta_{i}^{ \pm}\left(x_{i}, \cos \theta\right)\right]\right\} \\
& +\beta_{i}^{\text {non }-\tau}\left(x_{i}, \cos \theta\right) .
\end{aligned}
$$

In both cases, the corrected theoretical cross-sections depend on the two fit parameters $\left\langle P_{\tau}\right\rangle$ and $A_{p o l}^{F B}$. They also depend on $A_{F B}$, for which our measured value in the $Z^{0} \rightarrow \tau^{+} \tau^{-}$channel [5] at the appropriate centre-of-mass energy is used. The normalization factor in equation $19, \sigma_{i j}^{\prime}$, is the corrected cross-section calculated as the integral of equation 20 (or 21) over the kinematic variables. As various correction functions are slightly different for positive and negative helicity $\tau$ leptons, these normalization factors have small dependencies on $\left\langle P_{\tau}\right\rangle$ which are properly taken into account.

In performing the fit, the functions $h_{i(j)}^{ \pm}, \epsilon_{i(j)}, \beta_{i(j)}^{ \pm}, \mathcal{E}_{i(i j)}^{ \pm}$and $\beta_{i(i j)}^{\text {non- } \tau}$ as well as the normalization factors are calculated for each event. The $h_{i(j)}^{ \pm}$functions are obtained from the theoretical decay spectra corrected for radiative effects using large Monte Carlo $\tau^{+} \tau^{-}$event samples generated without detector simulation and corrected for resolution effects using response functions measured in data samples of $\mathrm{e}^{+} \mathrm{e}^{-} \rightarrow \mathrm{e}^{+} \mathrm{e}^{-}$events, low energy "single electron" events from highly radiative Bhabha scattering, and $\mathrm{e}^{+} \mathrm{e}^{-} \rightarrow \mu^{+} \mu^{-}$events. The $\epsilon_{i(j)}, \mathcal{E}_{i(i j)}^{ \pm}, \beta_{i(j)}^{ \pm}$ and $\beta_{i(i j)}^{\text {non }} \tau$ functions are parametrized in terms of simple functions, in most cases low order polynomials, using few parameters. The parameters are determined from fits to the pertinent Monte Carlo samples and subsequently corrected using appropriate control samples from the data. The uncertainties in the parameters, their correlations and the corrections are taken into account in the treatment of systematic errors which are discussed in detail below.

The maximum likelihood fit, applied to the $\tau \rightarrow \mathrm{e} \bar{\nu}_{e} \nu_{\tau}, \tau \rightarrow \mu \bar{\nu}_{\mu} \nu_{\tau}$ and $\tau \rightarrow \pi(\mathrm{K}) \nu_{\tau}$ decay channels yields the following results,

$$
\begin{aligned}
& \left\langle P_{\tau}\right\rangle=(-13.5 \pm 2.9(\text { stat })) \% \\
& \mathrm{~A}_{\text {pol }}^{F B}=(-11.0 \pm 3.5(\text { stat })) \%
\end{aligned}
$$

where the correlation between the two numbers is +0.03 . In order to evaluate the fit quality, we plot the $x$ distributions separately for each decay combination and compare it with the corresponding corrected theoretical curves (Figures 3, 4, 5). We remark that the complex behaviour of the theoretical distributions for the low $x$ region in the cases where both $\tau$ leptons decay to electrons or both decay to muons is understood to be caused by the requirements that remove two-photon events from the $\tau$-pair sample. The effect of the kaon threshold in the $\tau \rightarrow \pi(\mathrm{K}) \nu_{\tau}$ decays is also evident on these plots. The $x$ distributions for $\tau \rightarrow \mathrm{e} \bar{\nu}_{e} \nu_{\tau}, \tau \rightarrow \mu \bar{\nu}_{\mu} \nu_{\tau}$ and $\tau \rightarrow \pi(\mathrm{K}) \nu_{\tau}$ summing over all decays on the other $\tau$ jet are presented in Figure 6 . The normalization of the theoretical curves is obtained by a fit to the experimental distributions. The $\chi^{2}$ values and associated probabilities, which are calculated by comparing the maximum likelihood expectation to the binned data, are presented on the figures and show that the agreement 
between the experimental distributions and the theoretical curves is good. The hatched regions in the figures represent the background and the dotted lines are the uncorrected theoretical distributions. One can see that the background contributions are small, and the overall corrections to the theoretical curves, excluding the first and second bins in $x$, are smooth, and do not depend strongly on $x$. Recall, however, that only data with $x$ greater than 0.05 are included in the analysis. The fit quality is also demonstrated by presenting the polarization as a function of $\cos \theta$ in Figure 7 . For the purposes of this presentation, the data have been separated into five bins of $\cos \theta$ and a value of the polarization extracted for each bin using the maximum likelihood fit modified such that the $\cos \theta$ terms in equations 20 and 21 are ignored. The result of the maximum likelihood fit is represented as a solid curve in Figure 7.

Detailed discussions of each of the correction functions and the associated systematic uncertainties are presented in the following sections and are summarized in Table 2.

\begin{tabular}{||l|c|c||}
\hline \hline Source & $\begin{array}{c}\Delta\left\langle P_{\tau}\right\rangle \\
(\%)\end{array}$ & $\begin{array}{c}\Delta \mathrm{A}_{\text {pol }}^{F B} \\
(\%)\end{array}$ \\
\hline radiative effects & 0.2 & 0.0 \\
A $_{F B}$ and K threshold & 0.1 & 0.1 \\
calorimeter response & 0.5 & 0.1 \\
tracking response & 0.4 & 0.2 \\
cos $\theta$ and charge measurement & 0.0 & 0.2 \\
$\tau$-pair selection efficiency & 0.4 & 0.2 \\
$\tau$ decay identification & 1.8 & 0.2 \\
backg. from other $\tau$ decays & 0.7 & 0.1 \\
backg. from non- $\tau$ events & 0.7 & 0.2 \\
\hline Total & 2.2 & 0.5 \\
\hline \hline
\end{tabular}

Table 2: Summary of systematic uncertainties in the determination of $\left\langle P_{\tau}\right\rangle$ and $\mathrm{A}_{\text {pol }}^{F B}$ using $\tau \rightarrow \mathrm{e} \bar{\nu}_{e} \nu_{\tau}, \tau \rightarrow \mu \bar{\nu}_{\mu} \nu_{\tau}$ and $\tau \rightarrow \pi(\mathrm{K}) \nu_{\tau}$ channels.

\section{Radiative and threshold effects}

For a given helicity state, initial and final state radiation as well as radiation in the decay of the $\tau$ affect the spectra of the $\tau$ decay products. The radiative distortions to the spectrum for decay mode $i$ are taken into account using a radiative correction function, $r_{i}^{ \pm}\left(x_{i}\right)$. The function $r_{i}^{ \pm}\left(x_{i}\right)$ is determined from the ratio of the spectrum containing radiative effects to the spectrum where only the Born level cross-section is considered. A high statistics run of the KORALZ 4.0 Monte Carlo generator [14], without detector simulation, is used to create the radiatively corrected spectrum of the appropriate kinematic variable in each channel ${ }^{4}$. The ratio of distributions for positive and negative helicity are treated separately in order to disentangle kinematic distortions to the Born level spectrum caused by photonic radiative corrections to $\left\langle P_{\tau}\right\rangle$ or $A_{\text {pol }}^{F B}$. These latter effects are taken into account explicitly when interpreting the measurements as a determination of $\sin ^{2} \theta_{\text {eff }}^{\text {lept }}$.

\footnotetext{
${ }^{4}$ KORALZ 4.0 extends the KORALZ 3.8 treatment of final state bremsstrahlung to $\mathcal{O}\left(\alpha^{2}\right)$ (including exclusive exponentiation); calculates the decay radiation in the leptonic decays to $\mathcal{O}(\alpha)$; and decay radiation in all semileptonic decay modes in the leading logarithmic approximation.
} 
A potentially important uncertainty associated with these corrections arises from the fact that the QED $\mathcal{O}(\alpha)$ corrections to semi-leptonic $\tau$ decays are included only in the leading logarithmic approximation. The effect of this correction on the polarization asymmetries is quantified by comparing results using radiative correction functions, $r_{i}^{ \pm}\left(x_{i}\right)$, in which the radiation in the decay of the semi-leptonic modes has not been included to those for which it has been included. The difference in the results is used as a measure of the magnitude of the correction. The correction is estimated to have a systematic error of $5 \%$ of the correction[20] and, for the $\tau \rightarrow \pi(\mathrm{K}) \nu_{\tau}$ mode, introduces a negligible contribution to the error. The dominant contribution to the error on $r_{i}^{ \pm}\left(x_{i}\right)$ arises from the Monte Carlo statistics used to calculate $r_{i}^{ \pm}\left(x_{i}\right)$ while the treatment of initial and final state radiation in KORALZ introduces a negligible contribution to the error. The total error assigned to the radiative correction uncertainties on $\left\langle P_{\tau}\right\rangle$ and $\mathrm{A}_{\text {pol }}^{F B}$ are $0.2 \%$ and less than $0.1 \%$, respectively.

The effect of the threshold for $\tau$ decays to kaons (equation 15) introduces an error on $\left\langle P_{\tau}\right\rangle$ and $\mathrm{A}_{\text {pol }}^{F B}$ associated with the uncertainties in the $\tau$ branching ratios to pions and kaons and leads to errors of approximately $0.1 \%$. There is also a very small error (much less than $0.1 \%$ ) introduced from the measurement uncertainty on $A_{F B}[5]$ which enters the analysis (see equation 8).

\section{Detector resolution}

The distribution in $x$, after taking into account the radiative effects and the detector resolution, can be written as,

$$
h_{i}^{ \pm}\left(x_{i}\right)=C_{i} \int_{0}^{1}\left[f_{i}\left(x_{i}^{0}\right) \pm g_{i}\left(x_{i}^{0}\right)\right] r_{i}^{ \pm}\left(x_{i}^{0}\right) \mathcal{R}_{i}\left(x_{i}^{0}, x_{i}\right) d x_{i}^{0}
$$

where a normalization factor $C_{i}$ was introduced to assure that,

$$
\int_{0}^{\infty} h_{i}^{ \pm}\left(x_{i}\right) d x_{i}=1
$$

Here $\mathcal{R}_{i}\left(x_{i}^{0}, x_{i}\right)$ represents the response function which is the distribution of the measured $x_{i}$ for a given true $x_{i}^{0}$ and depends on the measured particle.

For electrons, $x$ is the electromagnetic energy in the $\tau$ jet normalized to the beam energy. The shape of $\mathcal{R}_{i}\left(x_{i}^{0}, x_{i}\right)$ is investigated using electron-pair events and is parametrized by a sum of Gaussians with various width, mean and normalization parameters. For the energy dependence of the width, electron-pair and single electron events from the data are used. In electron-pair events, the electrons are assumed to have the beam energy, whereas for single electrons the widths and means of the $E / p$ distributions at different energies are used. The contributions of momentum resolution and bremsstrahlung to the width are taken into account when determining the energy resolution. The overall energy scale of the ECAL is calibrated using electron-pair events in the data and has an uncertainty of $\pm 0.3 \%$. At lower energies, where the amount of energy which is lost in the material in front of the calorimeter is significant, data from $\tau \rightarrow \mathrm{e} \bar{\nu}_{e} \nu_{\tau}$ decays and single electrons are used to study the energy scale as described in Section 6 . The systematic error assigned to the response function includes contributions from the energy-loss uncertainty, ECAL calibration and the statistical errors on the parameters and their correlations. These contribute an error of $0.5 \%$ on $\left\langle P_{\tau}\right\rangle$ and $0.1 \%$ on $\mathrm{A}_{\text {pol }}^{F B}$.

For a muon and $\pi(\mathrm{K}), x$ is essentially the particle momentum normalized to the beam energy ${ }^{5}$. In order to investigate the shape of the response function, the $x$ measurements from

\footnotetext{
${ }^{5}$ For the $\tau \rightarrow \mu \bar{\nu}_{\mu} \nu_{\tau}$ analysis, $x$ also includes energy from photons in the $\tau$ jet.
} 
colinear $\mathrm{e}^{+} \mathrm{e}^{-} \rightarrow \mu^{+} \mu^{-}$events are used. For these events, the value of $x^{0}$ is assumed to be one and the shape is also parametrized by a sum of Gaussians. The energy dependence of the width parameters is determined from $\tau \rightarrow \mu \bar{\nu}_{\mu} \nu_{\tau}$ and $\tau \rightarrow \pi(\mathrm{K}) \nu_{\tau}$ decays modelled in the $\tau$-pair Monte Carlo sample. The statistical errors on the parameters as well as their correlations contribute to the systematic errors assigned to the response function. In addition, a possible dependence of the momentum measurement on $\cos \theta$, which would affect the extracted $A_{\text {pol }}^{F B}$ value, is considered. In order to investigate this effect, the momentum of muons from $\mu$-pair events was measured as function of $\cos \theta$. A slight dependence, $\Delta p / p=-(2.0 \pm 0.9) \cdot 10^{-3} \cdot \cos \theta$, is present. Introducing this dependence yields deviations in $\left\langle P_{\tau}\right\rangle$ and $A_{\text {pol }}^{F B}$ of around $0.2 \%$. As there is uncertainty in the momentum dependence of this effect, the polarization results are not modified but the full $0.2 \%$ is taken as a contribution to the systematic error. The dependence of the momentum resolution on $\cos \theta$ of the track introduces a contribution to the error of $0.3 \%$ and $0.02 \%$ on $\left\langle P_{\tau}\right\rangle$ and $A_{\text {pol }}^{F B}$, respectively. The total errors assigned to the understanding of the tracking chamber response are $0.4 \%$ and $0.2 \%$ for $\left\langle P_{\tau}\right\rangle$ and $A_{\text {pol }}^{F B}$, respectively.

Corrections due to $\tau^{-}$scattering angle resolution and charge misassignment are not included in the fit and the lack of such corrections potentially contributes to the uncertainty on $A_{\text {pol }}^{F B}$. Monte Carlo studies indicate that the resolution in the cosine of the scattering angle is 0.023 . The effect of this resolution on the extracted $A_{\text {pol }}^{F B}$ fit results is less than $0.1 \%$. The fit is sensitive mainly to the spectrum of forward and backward scattered particles rather than the accurate shape of the $\cos \theta$ distribution. The uncertainty on $A_{\text {pol }}^{F B}$ arising from a wrong charge assignment of the $\tau$ jets which results in a wrong $\operatorname{sign}$ of $\cos \theta$ is also less than $0.1 \%$.

\section{Tau-pair selection efficiency}

The efficiency of $\tau$-pair selection, $\mathcal{E}^{ \pm}$, is investigated using the $\tau$-pair Monte Carlo events by comparing the kinematic variable distributions before and after the selection requirements. This is done separately for events with positive or negative $\tau^{-}$helicity and for the various combinations of the decays of the two $\tau$ leptons. When both $\tau$ leptons decay via one of the modes considered here $(\mathrm{e}, \mu$ or $\pi(\mathrm{K}))$, the efficiency is parametrized as a function of the kinematic variables $x_{i}, x_{j}$ and $\cos \theta$. As mentioned above, the $\tau$-pair selection requirements against $\mu$-pair, electron-pair and two-photon events involve two and sometimes all three kinematic variables in a correlated manner and these are explicitly taken into account in the parametrization of the efficiency ${ }^{6}$. When only one $\tau$ decays to e, $\mu$ or $\pi(\mathrm{K})$ and the other $\tau$ is not identified, the selection efficiency has two components. The first accounts for the case when the other $\tau$ does not decay via e, $\mu$ or $\pi(\mathrm{K})$ whilst the second accounts for the case where it does decay via these channels. The first component is parametrized as a function of $x_{i}$ and $\cos \theta$, assuming that these two kinematic variables are not correlated. The second component, which arises from identification inefficiencies, can have correlations and they are taken into account.

The error coming from the $\tau$-pair selection efficiency includes a component caused by limited Monte Carlo statistics and another related to the resolution and scale uncertainties in the ECAL and tracking detector as previously discussed. The contributions from the $\tau$-pair selection efficiency to the overall uncertainty on $\left\langle P_{\tau}\right\rangle$ and $\mathrm{A}_{\text {pol }}^{F B}$ are $0.4 \%$ and $0.2 \%$, respectively.

\footnotetext{
${ }^{6}$ The other $\tau$-pair selection requirements do not introduce significant biases against the $\tau \rightarrow \mathrm{e} \bar{\nu}_{e} \nu_{\tau}, \tau \rightarrow \mu \bar{\nu}_{\mu} \nu_{\tau}$ or $\tau \rightarrow \pi(\mathrm{K}) \nu_{\tau}$ channels.
} 


\section{Efficiencies of the $\tau$ decay identification}

The efficiencies to identify the various $\tau$ decays are obtained in two steps. In the first step, the Monte Carlo events are used in order to obtain the efficiency distributions for each decay channel, separately for events with positive or negative $\tau^{-}$helicity. These distributions depend on the $x$ variables of the identified $\tau$ and on $\cos \theta$. However, some of the identification requirements, such as those designed to remove electron-pair and $\mu$-pair events, also introduce a dependence on the $x$ variable of the $\tau$ on the opposite side. This dependence is taken into account. In the second step events from control samples in the data are used to correct the Monte Carlo efficiency distributions for possible effects not modelled correctly in the detector simulation, as detailed below. The selection procedures of these control samples are largely independent of the $\tau$ decay identification requirements. The efficiencies obtained from those real data events are compared with those from Monte Carlo events of the same process which passed the same selection criteria. In this way, any bias due to the selection of the control sample itself is minimized.

For $\tau \rightarrow \mathrm{e} \bar{\nu}_{e} \nu_{\tau}$ decays, electron-pair events provide a control sample of high energy electrons whilst single electron events are used to control the region below $10 \mathrm{GeV}$. In order to investigate the muon detection efficiency we used $\mu$-pair events for high energy and $\gamma \gamma \rightarrow \mu^{+} \mu^{-}$events for low energy muons. In both cases, these control sample studies result in approximately $5 \%$ efficiency corrections which are approximately flat as a function of $x$. We use a linear parameterization of this slight dependence and assign a systematic error which accounts for the statistics of the control samples and the uncertainty in extrapolating between the low and high $x$ regions.

For single charged pions or kaons there are no clean non-tau control samples available over the momentum range of interest. Instead, decays of the $\tau$ into hadronic states containing neutral pions and a single charged hadron are used. For example, the $\pi(\mathrm{K})$ identification requirements that remove muons can be controlled using the $\tau \rightarrow \rho \nu_{\tau}$ sample since these requirements are not used in the $\tau \rightarrow \rho \nu_{\tau}$ identification. The resulting correction for these requirements is approximately flat, being equal to 0.99 , but can also be parametrized as a second order polynomial. We use the flat correction function, but investigate the effect of using the other alternative in the systematic study.

Requirements on the presampler signal and ECAL energy which is not associated with the charged track are used to remove backgrounds containing neutral pions[3] but these also introduce a significant bias. The reliability of the modelling of these requirements is studied using an alternative pion selection based on a low jet mass requirement. This sample has a larger background $(12 \%)$ but is clean enough to estimate the systematic dependence of the results on the standard requirements. The conclusion of this study is that the Monte Carlo modelling of the $x$ dependence of the efficiency is in excellent agreement with the data. This is verified using a subsample of the $\tau \rightarrow \rho \nu_{\tau}$ events in which the neutral pion is well separated from the track and with a sample of very high momenta $\tau \rightarrow \pi(\mathrm{K}) \nu_{\tau}$ events which has little background from the other hadronic decays. The statistical error on this control sample investigation yields systematic errors of $1.5 \%$ and $0.1 \%$ on $\left\langle P_{\tau}\right\rangle$ and $A_{\text {pol }}^{F B}$, respectively.

In order to remove the $\tau \rightarrow \mathrm{e} \bar{\nu}_{e} \nu_{\tau}$ background, the ratio of the ECAL energy associated with the track to the track momentum is required to be less than 0.8 . The $\tau \rightarrow \rho \nu_{\tau}$ control sample is used to demonstrate that this requirement introduces a negligible contribution to the overall systematic error.

These various efficiency corrections contribute systematic errors on $\left\langle P_{\tau}\right\rangle$ and $A_{\text {pol }}^{F B}$ of $1.8 \%$ and $0.2 \%$, respectively. 


\section{Background}

As seen from equations 20 and 21 , the correction for background contamination in channel $i$ is performed separately for background from other $\tau$ decay sources $\left(\beta_{i}^{ \pm}\right.$which depends on the $\tau$ helicity) and background from non- $\tau$ sources $\left(\beta_{i(j)}^{\text {non }} \tau\right)$. The first type of background is initially investigated using Monte Carlo events separately for positive and negative $\tau^{-}$helicity. For $\tau \rightarrow \mathrm{e} \bar{\nu}_{e} \nu_{\tau}$, the background level from other $\tau$ decays is $\approx 4.7 \%$, differing slightly between positive and negative $\tau^{-}$helicity events, and originating mainly from $\tau \rightarrow \pi(\mathrm{K}) \nu_{\tau}$ and $\tau \rightarrow \rho \nu_{\tau}$ decays. For $\tau \rightarrow \mu \bar{\nu}_{\mu} \nu_{\tau}$ this background is $\approx 1.5 \%$ coming from $\tau \rightarrow \pi(\mathrm{K}) \nu_{\tau}$ decays, and the background to $\tau \rightarrow \pi(\mathrm{K}) \nu_{\tau}$ is $\approx 7.2 \%$, mainly from $\tau \rightarrow \rho \nu_{\tau}, \tau \rightarrow \pi K^{0} \nu$ and $\tau \rightarrow \mathrm{e} \bar{\nu}_{e} \nu_{\tau}$ decays. The Monte Carlo expectations for these background levels are checked against the data by studying the $\tau$ jets that are identified in more than one decay channel and from studies of how electrons and muons in the electron-pair and $\mu$-pair control samples contaminate the pion sample. An error on the $\tau \rightarrow \pi \mathrm{K}^{0} \nu$ background is estimated by varying the Monte Carlo expectation by $\pm 100 \%$. A small $(<0.1 \%)$ additional contribution to the errors arising from the uncertainties in $\tau$ branching ratios has been included. These background sources contribute errors of $0.7 \%$ and $0.1 \%$ on $\left\langle P_{\tau}\right\rangle$ and $\mathrm{A}_{\text {pol }}^{F B}$, respectively.

Concerning non- $\tau$ background, the following sources are considered.

- $\mathrm{e}^{+} \mathrm{e}^{-} \rightarrow \mu^{+} \mu^{-}$. This source mainly contaminates events where one $\tau$ is identified as $\tau \rightarrow \mu \bar{\nu}_{\mu} \nu_{\tau}$ decay and the other as $\tau \rightarrow \pi(\mathrm{K}) \nu_{\tau}$ or when one $\tau$ is identified as a $\tau \rightarrow \mu \bar{\nu}_{\mu} \nu_{\tau}$ and the other is not identified, at the level of $0.4 \%$ and $1.3 \%$, respectively.

- $\mathrm{e}^{+} \mathrm{e}^{-} \rightarrow \mathrm{e}^{+} \mathrm{e}^{-} \mu^{+} \mu^{-}$. This source contributes only to events with two identified muons or one identified muon and an unidentified $\tau$, at the level of $1.6 \%$ and $0.3 \%$, respectively.

- $\mathrm{e}^{+} \mathrm{e}^{-} \rightarrow \mathrm{e}^{+} \mathrm{e}^{-}$. This source contributes background to events where only one $\tau$ decay is identified as e or $\pi(\mathrm{K})$ (and the other $\tau$ decay is unidentified) and events where both decays are identified as $\tau \rightarrow \mathrm{e} \bar{\nu}_{e} \nu_{\tau}$. The corresponding contamination levels are $0.3 \%, 0.2 \%$ and $0.3 \%$, respectively.

- $\mathrm{e}^{+} \mathrm{e}^{-} \rightarrow \mathrm{e}^{+} \mathrm{e}^{-} \mathrm{e}^{+} \mathrm{e}^{-}$. This source contaminates only events with one electron where the opposite $\tau$ decay is unidentified or two identified electrons, at the level of $0.3 \%$ and $2.6 \%$, respectively.

- $\mathrm{e}^{+} \mathrm{e}^{-} \rightarrow q \bar{q}$. The background from this source is negligible for all channels considered here.

The $\beta_{i(j)}^{\text {non }-\tau}\left(x_{i}, x_{j}, \cos \theta\right)$ correction functions for each source were initially determined using the corresponding Monte Carlo event samples. In practice the correlations between $x_{i(j)}$ and $\cos \theta$ are small and the $\beta_{i(j)}^{\text {non }} \tau$ functions factorize into products of simple functions. These were adjusted by factors obtained from comparisons between the data and Monte Carlo when the $\tau$ selection requirements designed to suppress these backgrounds were loosened. The contribution to the errors on $\left\langle P_{\tau}\right\rangle$ and $A_{\text {pol }}^{F B}$ from the non-tau background corrections are $0.7 \%$ and $0.2 \%$, respectively.

\section{Cross checks of the fitting method}

In order to check for potential biases in the fitting method, the analysis was performed on the $\tau$-pair Monte Carlo sample using resolution parameters extracted exclusively from Monte Carlo events. The input values for $\left\langle P_{\tau}\right\rangle$ and $\mathrm{A}_{\text {pol }}^{F B}$ are $-14.0 \%$ and $-9.9 \%$, respectively and the 
fit returns values of $(-13.9 \pm 1.1) \%$ and $(-8.9 \pm 1.3) \%$. A similar check was performed on the Monte Carlo sample of purely positive and purely negative helicity states and, again, there is no evidence for a bias in either $\left\langle P_{\tau}\right\rangle$ or $A_{\text {pol }}^{F B}$.

The data have also been classified into nine independent subsamples, corresponding to all possible combinations of both $\tau$ decays not involving an identified $\tau \rightarrow \rho \nu_{\tau}$ decay. When doing so, nine independent results for $\left\langle P_{\tau}\right\rangle$ and $\mathrm{A}_{\text {pol }}^{F B}$ are obtained. These are listed in Table 3 . The weighted means of these values are within $0.2 \%$ of the global fit results and the $\chi^{2}$ probabilities for the consistency between the nine results of $\left\langle P_{\tau}\right\rangle$ and $A_{\text {pol }}^{F B}$ are $17 \%$ and $82 \%$, respectively. When we perform this exercise on the Monte Carlo, there is no evidence for a bias in any of the nine subsamples.

\begin{tabular}{|c|c|c|c|c|c|c|c|c|}
\hline \multirow{2}{*}{\multicolumn{2}{|c|}{$\begin{array}{l}\text { Decay channel } \\
\text { combination } \\
\text { Global ML fit values }\end{array}$}} & \multicolumn{3}{|c|}{$\begin{array}{l}\left\langle P_{\tau}\right\rangle \\
(\%)\end{array}$} & \multicolumn{3}{|c|}{$\begin{array}{l}A_{p o l}^{F B} \\
(\%)\end{array}$} & $\begin{array}{c}\text { Number of } \\
\text { Events }\end{array}$ \\
\hline & & -13.5 & \pm & 2.9 & -11.0 & \pm & 3.5 & 12373 \\
\hline \multirow{2}{*}{\multicolumn{2}{|c|}{$\begin{array}{l}\tau \text { identified } \\
2 \tau \text { 's identified }\end{array}$}} & -17.5 & \pm & 3.5 & -10.2 & \pm & 4.3 & 9250 \\
\hline & & -5.5 & \pm & 5.0 & -12.6 & \pm & 5.9 & 3123 \\
\hline$\tau \rightarrow \mathrm{e} \bar{\nu}_{e} \nu_{\tau}$ & none & -24.7 & \pm & 8.9 & -17. & \pm & 10. & 3585 \\
\hline$\tau \rightarrow \mu \bar{\nu}_{\mu} \nu_{\tau}$ & none & -8.7 & \pm & 7.9 & -10.8 & \pm & 9.1 & 3897 \\
\hline$\tau \rightarrow \pi(\mathrm{K}) \nu_{\tau}$ & none & -18.4 & \pm & 4.4 & -8.1 & \pm & 5.5 & 1768 \\
\hline$\tau \longrightarrow \mathrm{e} \bar{\nu}_{e} \nu_{\tau}$ & $\tau \longrightarrow \mathrm{e} \bar{\nu}_{e} \nu_{\tau}$ & 9. & \pm & 16. & -29 & \pm & 18. & 565 \\
\hline$\tau \longrightarrow \mathrm{e} \bar{\nu}_{e} \nu_{\tau}$ & $\tau \rightarrow \mu \bar{\nu}_{\mu} \nu_{\tau}$ & -16 & \pm & 10. & 3. & \pm & 12. & 1288 \\
\hline$\tau \rightarrow \mathrm{e} \bar{\nu}_{e} \nu_{\tau}$ & $\tau \rightarrow \pi(\mathrm{K}) \nu_{\tau}$ & -9.5 & \pm & 9.7 & -23 & \pm & 12. & 346 \\
\hline$\tau \longrightarrow \mu \bar{\nu}_{\mu} \nu_{\tau}$ & $\tau \rightarrow \mu \bar{\nu}_{\mu} \nu_{\tau}$ & 7. & \pm & 24. & 2. & \pm & 27. & 484 \\
\hline$\tau \rightarrow \mu \bar{\nu}_{\mu} \nu_{\tau}$ & $\tau \rightarrow \pi(\mathrm{K}) \nu_{\tau}$ & 6.1 & \pm & 9.0 & -13. & \pm & 11. & 385 \\
\hline$\tau \rightarrow \pi(\mathrm{K}) \nu_{\tau}$ & $\tau \rightarrow \pi(\mathrm{K}) \nu_{\tau}$ & -29 & \pm & 20. & -21 & \pm & 26. & 55 \\
\hline \multicolumn{2}{|l|}{ Average } & -13.3 & \pm & 2.9 & -11.1 & \pm & 3.4 & \\
\hline \multicolumn{2}{|c|}{$\begin{array}{l}\chi^{2} / D O F \\
\chi^{2} \text { probability }(\%)\end{array}$} & \multicolumn{3}{|c|}{$\begin{array}{c}11.6 / 8 \\
17.2 \\
\end{array}$} & \multicolumn{3}{|c|}{$\begin{array}{l}4.4 / 8 \\
81.6 \\
\end{array}$} & \\
\hline
\end{tabular}

Table 3: $\tau$ polarization results for the global fit and for each of the nine independent subsamples, corresponding to all possible combinations of identified $\tau \rightarrow \mathrm{e} \bar{\nu}_{e} \nu_{\tau}, \tau \rightarrow \mu \bar{\nu}_{\mu} \nu_{\tau}$ or $\tau \rightarrow \pi(\mathrm{K}) \nu_{\tau}$ decays. The average of these and the $\chi^{2}$ for this set is also given. Also quoted are the results of separate global fits to those events in which both $\tau$ decays have been identified and those events in which only one $\tau$ decay has been identified.

The results were also checked by dividing the $\cos \theta$ range into five bins and calculating the $\tau$ polarization in each bin separately using the maximum likelihood fit. Using $A_{F B}=$ $1.2 \%$, which is the OPAL result [5] for the $Z^{0}$ peak, we obtained $\left\langle P_{\tau}\right\rangle=(-13.9 \pm 2.9) \%$ and $\mathrm{A}_{\text {pol }}^{F B}=(-10.8 \pm 3.5) \%$ in excellent agreement with the maximum likelihood results. The fit $\chi^{2}$ is 0.7 for three degrees of freedom and the correlation between $\left\langle P_{\tau}\right\rangle$ and $\mathrm{A}_{\text {pol }}^{F B}$ is +0.03 .

For comparison with previous measurements, we list the results for each decay channel in Table 5 separately. One should note, however, that the results for the $\tau \rightarrow \mathrm{e} \bar{\nu}_{e} \nu_{\tau}, \tau \rightarrow \mu \bar{\nu}_{\mu} \nu_{\tau}$ and $\tau \rightarrow \pi(\mathrm{K}) \nu_{\tau}$ decays in this table cannot be interpreted as independent polarization measurements because the same $\tau$-pair event can contribute twice. For our selected $\tau$ decays, failure to include these correlations would lead to a $7 \%$ and $9 \%$ artificial reduction in the statistical errors on $\left\langle P_{\tau}\right\rangle$ and $\mathrm{A}_{\text {pol }}^{F B}$, respectively. 
As an additional cross-check, we have performed an independent polarization analysis which is similar to that described in our previous publication [3]. It consists of a set of least squares fits to background-subtracted $\tau \rightarrow \mathrm{e} \bar{\nu}_{e} \nu_{\tau}, \tau \rightarrow \mu \bar{\nu}_{\mu} \nu_{\tau}$ and $\tau \rightarrow \pi(\mathrm{K}) \nu_{\tau}$ spectra applied separately to data in the forward and backward hemispheres. The theoretical spectra are corrected for efficiency, resolution, radiation and threshold effects. The background and efficiencies as predicted by the Monte Carlo simulation are corrected using data control samples in a manner similar to that used in the maximum likelihood analysis. This analysis does not take into account the correlations between the two $\tau$ leptons or the polarization dependence of the background. The results are in good agreement with those obtained from the maximum likelihood fit which is modified to ignore the correlations between the two $\tau$ leptons. The global values for $\left\langle P_{\tau}\right\rangle$ and $\mathrm{A}_{\text {pol }}^{F B}$ from the least squares analysis are both within $0.5 \%$ of the results from the maximum likelihood fit.

\section{Summary of maximum likelihood results}

Table 2 summarizes the systematic errors in the maximum likelihood analysis. Each error listed in Table 2 is a combined result of several related contributions and as there are no correlations between entries on different rows of the table, they are combined in quadrature to give the overall systematic error listed in the last row of Table 2. Thus the values of $\left\langle P_{\tau}\right\rangle$ and $A_{\text {pol }}^{F B}$ using the $\tau \rightarrow \mathrm{e} \bar{\nu}_{\epsilon} \nu_{\tau}, \tau \rightarrow \mu \bar{\nu}_{\mu} \nu_{\tau}$ and $\tau \rightarrow \pi(\mathrm{K}) \nu_{\tau}$ decay channels are measured to be

$$
\begin{aligned}
& \left\langle P_{\tau}\right\rangle=(-13.5 \pm 2.9 \pm 2.2) \% \\
& \mathrm{~A}_{\text {pol }}^{F B}=(-11.0 \pm 3.5 \pm 0.5) \%
\end{aligned}
$$

where the first error is statistical and the second systematic.

The dominant contribution to the systematic error on $\left\langle P_{\tau}\right\rangle$ derives from the uncertainties in the efficiency of the $\tau$ decay mode identification. For the most part these arise from the limited statistics of the control samples, and in particular, the $\tau \rightarrow \rho \nu_{\tau}$ sample used to correct the efficiency of $\tau \rightarrow \pi(\mathrm{K}) \nu_{\tau}$ identification. Although there is no single dominant systematic

error on $\mathrm{A}_{\text {pol }}^{F B}$ there are a number of significant contributions whose magnitudes depend on the control sample data sizes used in their evaluation. Therefore, one can expect the systematic errors to decrease as more data is collected.

\section{$6 \quad\left\langle P_{\tau}\right\rangle$ and $\mathbf{A}_{\text {pol }}^{F B}$ from $\tau \rightarrow \rho \nu_{\tau}$ decays}

For the analysis of the $\tau \rightarrow \rho \nu_{\tau}$ decay, $\left\langle P_{\tau}\right\rangle$ and $\mathrm{A}_{\text {pol }}^{F B}$ are determined from a two-stage fit which is performed separately for the two $\tau \rightarrow \rho \nu_{\tau}$ selections. The data satisfying a $\tau \rightarrow \rho \nu_{\tau}$ selection are divided into five equal sized bins in $\cos \theta$. In the first stage of the fit, the average $\tau$ polarization in each $\cos \theta$ bin is independently determined by fitting for $\left\langle P_{\tau}\right\rangle_{\theta}$ in a manner described below. The second stage consists of extracting the $\left\langle P_{\tau}\right\rangle$ and $A_{\text {pol }}^{F B}$ parameters in a least squares fit of the $\left\langle P_{\tau}\right\rangle_{\theta}$ dependence on $\cos \theta$, as given by equation 8 , using the $\left\langle P_{\tau}\right\rangle_{\theta}$ measurements obtained in the first stage.

For the first stage, the data in a given $\cos \theta$ bin are divided into bins of $\cos \theta^{*}$ and $\cos \psi$ which are defined in Section 2. The number of expected events in each bin is determined by integrating the joint distribution in $\left(\cos \theta^{*}, \cos \psi\right)$ over the $\cos \theta^{*}$ and $\cos \psi$ bin and correcting for detector response and background effects. 
The expected number of events in $\left(\cos \theta^{*}, \cos \psi\right)$ bin $(i, j), N_{i j}$, is expressed as

$$
N_{i j}(\theta)=\frac{1}{2}\left(1+\left\langle P_{\tau}\right\rangle_{\theta}\right) N_{i j}^{+}(\theta)+\frac{1}{2}\left(1-\left\langle P_{\tau}\right\rangle_{\theta}\right) N_{i j}^{-}(\theta)
$$

where

$$
N_{i j}^{ \pm}(\theta)=\frac{\epsilon_{i j}^{ \pm}(\theta) r_{i j}^{ \pm}}{\left(1-\beta_{i j}^{ \pm}(\theta)\right)} W^{ \pm}\left(\cos \theta^{*}, \cos \psi_{j}\right)
$$

The superscript refers to the $\tau^{-}$helicity state and the symbol $\epsilon_{i j}$ represents the efficiency for selecting a $\tau \rightarrow \rho \nu_{\tau}$ candidate in bin $\left(\cos \theta^{*}{ }_{i}, \cos \psi_{j}\right)$ and includes both the $\tau$-pair and $\tau \rightarrow \rho \nu_{\tau}$ selection efficiencies. The background fraction is represented by $\beta_{i j}$ and corrections for radiation by $r_{i j}$. The integral of the joint distribution in $\left(\cos \theta^{*}, \cos \psi\right)$ is represented by $W\left(\cos \theta^{*}, \cos \psi_{j}\right)$. The radiative correction function, $r_{i j}$, is obtained from a high statistics sample of KORALZ 4.0 Monte Carlo events.

The distributions in $\cos \theta^{*}$ for different regions of $\cos \psi$ for the $\tau \rightarrow \rho \nu_{\tau}$ selection I and II data are shown in Figures 8 and 9 where the $\cos \theta$ bins have been combined. The parameters extracted from the two analyses are:

$$
\begin{array}{rll}
\text { Sample I } & \left\langle P_{\tau}\right\rangle=(-15.9 \pm 3.0(\text { stat })) \% ; & \mathrm{A}_{\text {pol }}^{F B}=(-6.3 \pm 3.5(\text { stat })) \% \\
\text { Sample II } & \left\langle P_{\tau}\right\rangle=(-15.5 \pm 2.9(\text { stat })) \% ; & \mathrm{A}_{\text {pol }}^{F B}=(-7.7 \pm 3.3(\text { stat })) \%
\end{array}
$$

where only the statistical errors are given. We present the measured $\left\langle P_{\tau}\right\rangle_{\theta}$ as a function of $\cos \theta$ for both selections in Figure 10 . The $\chi^{2}$ value of the fit of the $\left\langle P_{\tau}\right\rangle_{\theta}$ dependence on $\cos \theta$ is 0.9 for sample I and 1.4 for sample II for three degrees of freedom. The values of the $\chi^{2}$ for the fits in each $\cos \theta$ bin range between 14 and 22 for selection I and between 18 and 26 for selection II where the number of degrees of freedom in each case is 24 .

The results from the two analyses are combined, taking into account the statistical correlation between the two sets of results $(37 \%)$, which is determined by analysing the common event sample, to produce an overall $\tau \rightarrow \rho \nu_{\tau}$ result of

$$
\begin{aligned}
& \left\langle P_{\tau}\right\rangle=(-15.7 \pm 2.4(\text { stat })) \% \\
& A_{\text {pol }}^{F B}=(-7.1 \pm 2.8(\text { stat })) \% .
\end{aligned}
$$

The $\left\langle P_{\tau}\right\rangle$ as a function of $\cos \theta$ for the combined $\tau \rightarrow \rho \nu_{\tau}$ data is shown in Figure $10 \mathrm{c}$. The correlation between the two parameters is +0.003 .

Discussions of the contributions to the systematic uncertainties are presented in the following sections and are summarized for both selections in Table 4.

\section{Radiative Effects}

A small contribution to the systematic error arising from the treatment of radiative effects is evaluated in a manner analogous to that described in Section 5. An analysis using radiative correction functions in which the radiation in the decay of the semi-leptonic modes has not been included is compared to that in which it has been included with the difference indicating the magnitude of the correction. Following reference [21], the uncertainty on the correction is estimated to be on the order of $1 / \ln \left(m_{\tau} / m_{\rho}\right)$ of the correction. Therefore, the difference, multiplied by $1 / \ln \left(m_{\tau} / m_{\rho}\right)$, is assigned as the uncertainty arising from the decay radiation treatment. 


\section{Detector response}

The uncertainty in the modelling of the resolution of the calorimeter is estimated by increasing the resolution in the Monte Carlo by $2 \%$ of the measured energy and then re-evaluating the efficiency functions. As previously mentioned, the overall energy scale of the ECAL is calibrated using electron-pair events in the data and is estimated to be correct to $0.3 \%$ of the measured energy. The effect of this uncertainty on the polarization measurements is estimated by varying the energy scale by $\pm 0.3 \%$ and taking the corresponding change in the measurement as an error. In addition to this effect, there is a small discrepancy between the energy scale in the data and Monte Carlo at lower energies as revealed in studies using $\tau \rightarrow \mathrm{e} \bar{\nu}_{e} \nu_{\tau}$ decays and single electrons where the track momentum provides the reference electron energy. This discrepancy, which is negligible above $5-10 \mathrm{GeV}$, increases to $2 \%$ at $2 \mathrm{GeV}$. It is a reflection of the uncertainty in the proportion of energy which is deposited in the material in front of the calorimeter as described in the Monte Carlo simulation. An error is assessed for this effect by applying an energy dependent variation of the energy scale which corresponds to the observed discrepancy between data and Monte Carlo.

The uncertainty in the energy scale of the tracking detector is investigated using high momentum tracks in $Z^{0} \rightarrow \mu^{+} \mu^{-}$events and low momentum tracks from pions of $\mathrm{K}_{\mathrm{S}}^{0}$ decays in multihadronic $\mathrm{Z}^{0}$ decays. The systematic error arising from this uncertainty is negligible. The uncertainty on the resolution as determined from the $\mathrm{Z}^{0} \rightarrow \mu^{+} \mu^{-}$events, however, introduces a $0.5 \%$ contribution to the systematic uncertainty. Systematic errors related to photon conversions in the material of the tracking chambers are negligible.

\section{Background}

The correction factors for the background in each bin in $\left(\cos \theta^{*}, \cos \psi\right)$ are determined using the Monte Carlo simulation. There are several contributions to the systematic error of the polarization arising from uncertainties in the modelling of the background. One class of error is caused by the $\tau$ branching ratio uncertainties. The contributions from these are assessed by varying the assumed branching ratios for each decay mode by plus or minus one standard deviation of the world average as determined in reference [8] and quoting the corresponding change in $\left\langle P_{\tau}\right\rangle$ and $A_{\text {pol }}^{F B}$ as the errors on these quantities. Another source of uncertainty in the background is related to the ability of the Monte Carlo to simulate the response of the detector to the $\tau$ decay modes other than the $\tau \rightarrow \rho \nu_{\tau}$ mode. This source is assumed to be accounted for in the variation of the energy scale and resolution of the calorimeter and tracking detector as described above.

As the $\tau \rightarrow \mathrm{a}_{1} \nu_{\tau}$ decay forms a significant background in the $\tau \rightarrow \rho \nu_{\tau}$ sample it is necessary to account for uncertainties in the modelling of the $\mathrm{a}_{1}$ itself. The $\mathrm{a}_{1}$ mass and width are varied by $\pm 25 \mathrm{MeV}$ and $\pm 100 \mathrm{MeV}$, respectively and the changes in the polarization results induced by these changes are quoted as the uncertainties arising from the lack of knowledge of $a_{1}$. Uncertainties from the modelling of $\tau \rightarrow \pi \geq 3 \pi^{0}$ are negligible.

\section{Miscellaneous systematic errors and other cross checks}

The Monte Carlo is used to estimate the bin-by-bin efficiencies and purities for positive and negative helicity states separately. The error arising from the limited Monte Carlo statistics is $1.2 \%$ for the $\left\langle P_{\tau}\right\rangle$ measurement and $1.5 \%$ for $\mathrm{A}_{\text {pol }}^{F B}$. This includes contributions of the Monte Carlo statistics to the radiation corrections. There is also a very small error introduced from 
the measurement uncertainty on $A_{F B}$ which enters the analysis (see equation 8).

Additional systematic studies have been performed to cross check the analyses. These included verifying the stability of the results when modifying different aspects of the $\tau \rightarrow \rho \nu_{\tau}$ selection criteria such as the fiducial acceptance; minimum energy thresholds on tracks and clusters; requirements on what constitutes a well measured track and neutral cluster; track-cluster association criteria; and details of the two clustering algorithms. The data were also analysed by performing a least squares fit to the data of a linear combination of positive and negative helicity distributions of the fully simulated Monte Carlo events. The results are consistent with the quoted values but have a larger Monte Carlo statistical error. This same type of analysis was used to investigate any potential dependence of the extracted polarization results on the reconstructed $\rho$ mass by dividing the sample into three bins of reconstructed $\rho$ mass. The $\chi^{2}$ values for the three measurements were 0.84 and 1.85 for $\left\langle P_{\tau}\right\rangle$ and $A_{\text {pol }}^{F B}$, respectively, indicating that there is no evidence for such a dependence. We have also looked for biases in the polarization extraction technique using Monte Carlo samples of purely positive and purely negative helicity events and have found no evidence for such biases.

\begin{tabular}{||l|c|c||c|c||}
\hline \hline \multirow{2}{*}{ Source } & \multicolumn{2}{|c||}{$\begin{array}{c}\Delta\left\langle P_{\tau}\right\rangle \\
(\%)\end{array}$} & \multicolumn{2}{c||}{$\Delta A_{\text {pol }}^{F B}$} \\
& \multicolumn{2}{|c||}{$(\%)$} \\
\cline { 2 - 5 } & Selection I & Selection II & Selection I & Selection II \\
\hline Radiative effects & 0.1 & 0.1 & 0.0 & 0.1 \\
calorimeter response & 0.2 & 0.5 & 0.2 & 0.2 \\
tracking response & 0.3 & 0.5 & 0.0 & 0.6 \\
Background & 0.6 & 0.4 & 0.0 & 0.1 \\
Monte Carlo Statistics & 1.2 & 1.2 & 1.5 & 1.4 \\
A & 0.0 & 0.0 & 0.1 & 0.1 \\
Total & 1.4 & 1.5 & 1.5 & 1.5 \\
\hline \hline
\end{tabular}

Table 4: Summary of systematic uncertainties in the determination of $\left\langle P_{\tau}\right\rangle$ and $A_{\text {pol }}^{F B}$ using the $\tau \rightarrow \rho \nu_{\tau}$ decay channel.

\section{Summary of the $\tau \rightarrow \rho \nu_{\tau}$ results}

Table 4 summarizes the different systematic errors relevant to the $\tau \rightarrow \rho \nu_{\tau}$ analysis. The various contributions to the systematic error are independent and therefore summed in quadrature in order to give a total systematic error. The analysis of the data using selection I has a total systematic error of $\pm 1.4 \%$ on $\left\langle P_{\tau}\right\rangle$ and $\pm 1.5 \%$ on $A_{\text {pol }}^{F B}$. For selection II the corresponding errors are $\pm 1.5 \%$ and $\pm 1.5 \%$, respectively. The systematic errors of the two analyses are assumed to be $100 \%$ correlated and are combined accordingly. The overall $\tau \rightarrow \rho \nu_{\tau}$ results are:

$$
\begin{aligned}
& \left\langle P_{\tau}\right\rangle=(-15.7 \pm 2.4 \pm 1.5) \% \\
& A_{\text {pol }}^{F B}=(-7.1 \pm 2.8 \pm 1.5) \%
\end{aligned}
$$

where the first error is statistical and the second systematic. 


\section{Summary: combined results and interpretation}

A summary of the measurements from all channels is presented in Table 5. The results are quoted for $\sqrt{s}=m_{Z}$. A very small correction which takes into account the fact that some data were collected off the peak of the $Z^{0}$ resonance is made. The final result, taking into account all correlations is:

$$
\begin{aligned}
\left\langle P_{\tau}\right\rangle & =(-14.9 \pm 1.9 \pm 1.3) \% \\
\mathrm{~A}_{\text {pol }}^{F B} & =(-8.9 \pm 2.2 \pm 0.9) \% .
\end{aligned}
$$

In evaluating the combined systematic error, those contributions from the momentum and energy scale, decay radiation and $A_{F B}$ uncertainties are assumed to be $100 \%$ correlated whereas all other sources are taken to be uncorrelated.

The $\left\langle P_{\tau}\right\rangle$ as a function of $\cos \theta$ for the combined data is shown in Figure 11. The correlation between the two parameters in the combined result is +0.02 .

These measurements are consistent with our previous measurement [3] and those of the other LEP collaborations [22, 23, 24].

\begin{tabular}{||l|c|c||}
\hline \hline Decay Channel & $\begin{array}{c}\left\langle P_{\tau}\right\rangle \\
(\%)\end{array}$ & $\begin{array}{c}\mathrm{A}_{\text {pol }}^{F B} \\
(\%)\end{array}$ \\
\hline$\tau \rightarrow \mathrm{e} \bar{\nu}_{e} \nu_{\tau}$ & $-8.5 \pm 5.8 \pm 4.5$ & $-10.4 \pm 6.6 \pm 1.3$ \\
$\tau \rightarrow \mu \bar{\nu}_{\mu} \nu_{\tau}$ & $-8.0 \pm 5.4 \pm 3.3$ & $-10.1 \pm 6.2 \pm 1.3$ \\
$\tau \rightarrow \pi(\mathrm{K}) \nu_{\tau}$ & $-14.3 \pm 3.7 \pm 3.0$ & $-10.9 \pm 4.5 \pm 0.8$ \\
\hline Global ML fit & $-13.5 \pm 2.9 \pm 2.2$ & $-11.0 \pm 3.5 \pm 0.5$ \\
\hline \hline$\tau \rightarrow \rho \nu_{\tau}$ & $-15.7 \pm 2.4 \pm 1.5$ & $-7.1 \pm 2.8 \pm 1.5$ \\
\hline \hline All channels combined & $-14.9 \pm 1.9 \pm 1.3$ & $-8.9 \pm 2.2 \pm 0.9$ \\
\hline
\end{tabular}

Table 5: Tau polarization results. Note that the individual results quoted for the $\tau \rightarrow \mathrm{e} \bar{\nu}_{e} \nu_{\tau}, \tau \rightarrow \mu \bar{\nu}_{\mu} \nu_{\tau}$ and $\tau \rightarrow \pi(\mathrm{K}) \nu_{\tau}$ channels assume, incorrectly, that there are no correlations between the $\tau$ leptons. These correlations are fully taken into account in the global maximum likelihood fit result. The results quoted for the $\tau \rightarrow \rho \nu_{\tau}$ decay and the combined results account for the correlations in a manner described in the text.

These results can be expressed in terms of $\mathcal{A}_{\tau}$ and $\mathcal{A}_{e}$ of equation 9 :

$$
\begin{aligned}
& \mathcal{A}_{\tau}=0.153 \pm 0.019 \pm 0.013 \\
& \mathcal{A}_{e}=0.122 \pm 0.030 \pm 0.012
\end{aligned}
$$

where ZFITTER [25] has been used to correct for the effects of the photon propagator, photon$\mathrm{Z}^{0}$ interference and photonic radiative corrections. Within the context of the Standard Model these can be interpreted as measurements of

$$
\begin{aligned}
& \hat{\mathrm{g}}_{v}^{\tau} / \hat{\mathrm{g}}_{a}^{\tau}=0.077 \pm 0.012 \\
& \hat{\mathrm{g}}_{v}^{e} / \hat{\mathrm{g}}_{a}^{e}=0.062 \pm 0.016
\end{aligned}
$$

where the errors include both statistical and systematic contributions. The agreement between these two values indicate that the data are consistent with the hypothesis of lepton universality. If universality is assumed, these results can be averaged to give

$$
\sin ^{2} \theta_{\mathrm{eff}}^{\text {lept }}=0.2321 \pm 0.0023 .
$$




\section{Acknowledgements}

We thank Z. Wass for many helpful discussions. It is a pleasure to thank the SL Division for the efficient operation of the LEP accelerator, the precise information on the absolute energy, and their continuing close cooperation with our experimental group. In addition to the support staff at our own institutions we are pleased to acknowledge the

Department of Energy, USA,

National Science Foundation, USA,

Particle Physics and Astronomy Research Council, UK,

Natural Sciences and Engineering Research Council, Canada,

Fussefeld Foundation,

Israel Ministry of Science,

Israel Science Foundation, administered by the Israel Academy of Science and Humanities,

Minerva Gesellschaft,

Japanese Ministry of Education, Science and Culture (the Monbusho) and a grant under the Monbusho International Science Research Program,

German Israeli Bi-national Science Foundation (GIF),

Direction des Sciences de la Matière du Commissariat à l'Energie Atomique, France,

Bundesministerium für Forschung und Technologie, Germany,

National Research Council of Canada,

A.P. Sloan Foundation and Junta Nacional de Investigação Científica e Tecnológica, Portugal.

\section{References}

[1] S.L. Glashow, Nucl. Phys. 22 (1961) 579;

S. Weinberg, Phys. Rev. Lett. 19 (1967) 1264;

A. Salam, Elementary Particle Theory, Ed. N. Svartholm, Stockholm, Almquist and Wiksell (1968) 367.

[2] S. Jadach and Z. Wass in Z Physics at LEP1, CERN 89-08, edited by G. Altarelli et al., Vol. 1 (1989) 235.

[3] OPAL Collab., G. Alexander et al., Phys. Lett. B266 (1991) 201.

[4] M. Consoli, W. Hollik and F. Jegerlehner in Z Physics at LEP1, CERN 89-08, edited by G. Altarelli et al., Vol. 1 (1989) 7.

[5] OPAL Collab., M.Z. Akrawy et al., Phys. Lett. B240 (1990) 497;

G. Alexander et al., Z. Phys. C52 (1991) 175;

P.D. Acton et al., Z. Phys. C58 (1993) 219;

R. Akers et al., Z. Phys. C61 (1994) 19.

[6] Y.S. Tsai, Phys. Rev. D4 (1971) 2821.

[7] A. Rougé, Workshop on Tau Lepton Physics (Orsay, 1990) eds M. Davier and B. JeanMarie, (Editions Frontieres, 1991) 213.

[8] Particle Data Group, K. Hikasa et al., Review of Particle Properties, Phys. Rev. D 45 (1992) 1. 
[9] K. Hagiwara, A.D. Martin and D. Zeppenfeld, Phys. Lett. B235 (1990) 198.

[10] OPAL Collab., K. Ahmet et al., Nucl. Instr. and Meth. A305 (1991) 275.

[11] M. Arignon et al., Nucl. Instr. and Meth. A313 (1992) 103.

[12] J. Allison et al., Nucl. Inst. and Meth. A317 (1992) 47.

[13] R. Brun, F. Bruyant, M. Maire, A. C. McPherson, and P. Zanarini, GEANT3, CERN DD/EE/84-1 (1987).

[14] S. Jadach, B.F.L Ward and Z. Wạs, Comp. Phys. Comm. 66 (1991) 276 (KORALZ 3.8);

S. Jadach, J.H. Kühn and Z. Wạs, Comp. Phys. Comm. 64 (1991) 275;

S. Jadach, B.F.L Ward and Z. Wa̧s, Comp. Phys. Comm. 79 (1994) 503 (KORALZ 4.0).

[15] T. Sjöstrand, Comp. Phys. Comm. 39 (1986) 347;

M. Bengtsson and T. Sjöstrand, Comp. Phys. Comm. 43 (1987) 367;

M. Bengtsson and T. Sjöstrand, Nucl. Phys. B289 (1987) 810 (JETSET).

[16] OPAL Collab., P. Acton et al., Z. Phys. C58 (1993) 387.

[17] M. Bohm, A. Denner and W. Hollik, Nucl. Phys. B304 (1988) 687;

F.A. Berends, R. Kleiss and W. Hollik, Nucl. Phys. B304 (1988) 712.

[18] R. Battacharya, J. Smith and G. Grammer, Phys. Rev. D15 (1977) 3267;

J. Smith, J.A.M. Vermaseren and G. Grammer, Phys. Rev. D15 (1977) 3280.

[19] OPAL Collab., R. Akers et al., Phys. Lett. B328 (1994) 207.

[20] Z. Wąs, private communication;

M. Finkemeier, Ph.D. Thesis, University of Karlsruhe (1994).

[21] E. Barberio, B. van Eijk and Z. Wạs, Comp. Phys. Comm. 66 (1991) 115.

[22] ALEPH Collab., D. Decamp et al., Phys. Lett. B265 (1991) 430;

ALEPH Collab., D. Buskulic et al., Z. Phys. C59 (1993) 369.

[23] DELPHI Collab., P. Abreu et al., Z. Phys. C55 (1992) 555.

[24] L3 Collab., O. Adriani et al., Phys. Lett. B294 (1992) 466.

[25] D. Bardin, et al., CERN-TH. 6443/92 (1992) (ZFITTER). 

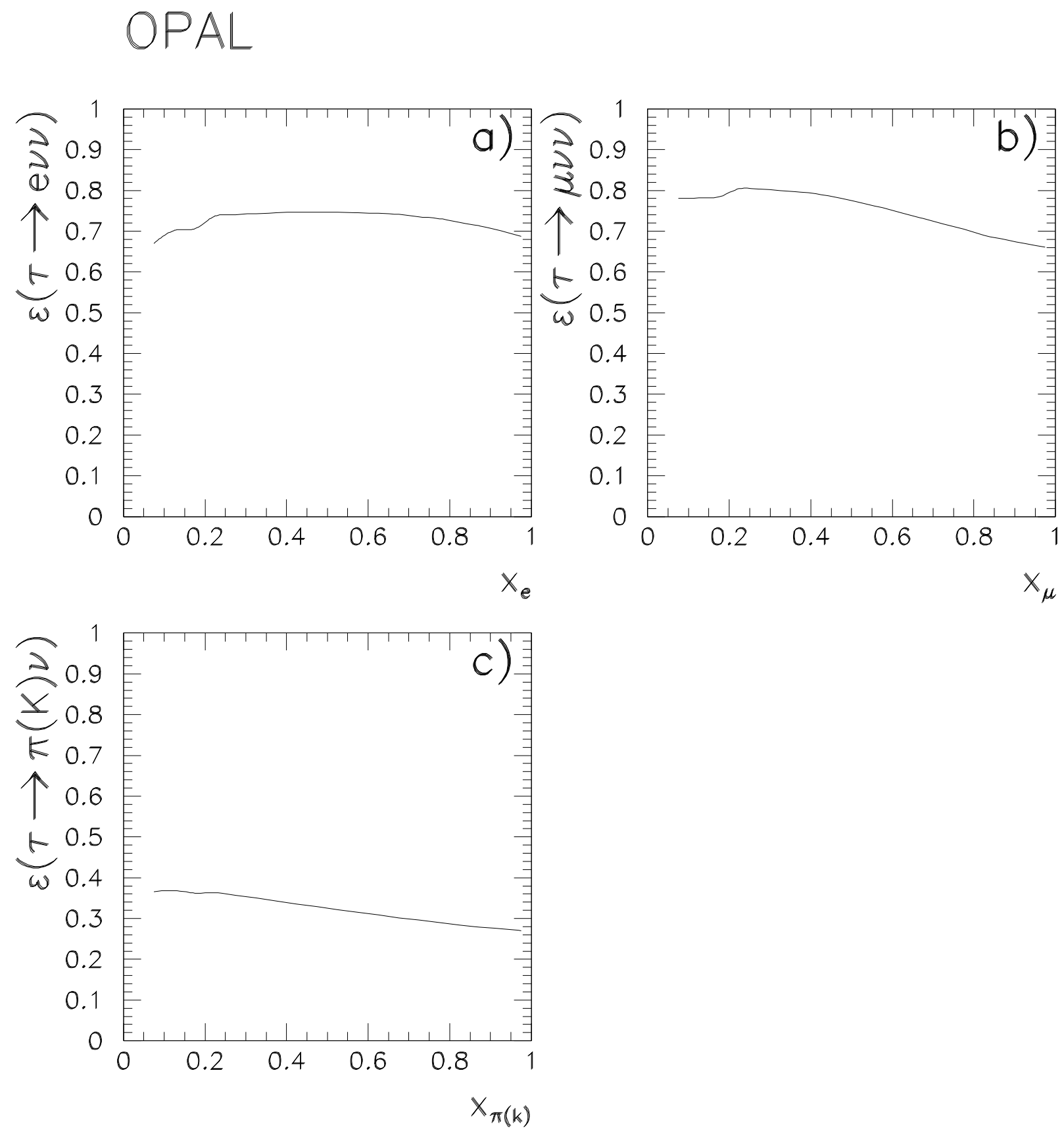

Figure 1: Global efficiencies as a function of $x$ for (a) $\tau \rightarrow \mathrm{e} \bar{\nu}_{e} \nu_{\tau}(\mathrm{b}) \tau \rightarrow \mu \bar{\nu}_{\mu} \nu_{\tau}$ and $(\mathrm{c}) \tau \rightarrow \pi(\mathrm{K}) \nu_{\tau}$ decays. The efficiencies include the $\tau$-pair selection efficiency and are after making the fiducial cut: $|\cos \theta|<0.68$. The drop in efficiency in the low $x$ region of $(a)$ and $(b)$ is caused by requirements designed to remove two-photon, electron-pair and $\mu$-pair events. Note that the requirement that removes candidates having $x<0.05$ is evident on these plots. 
OPAL
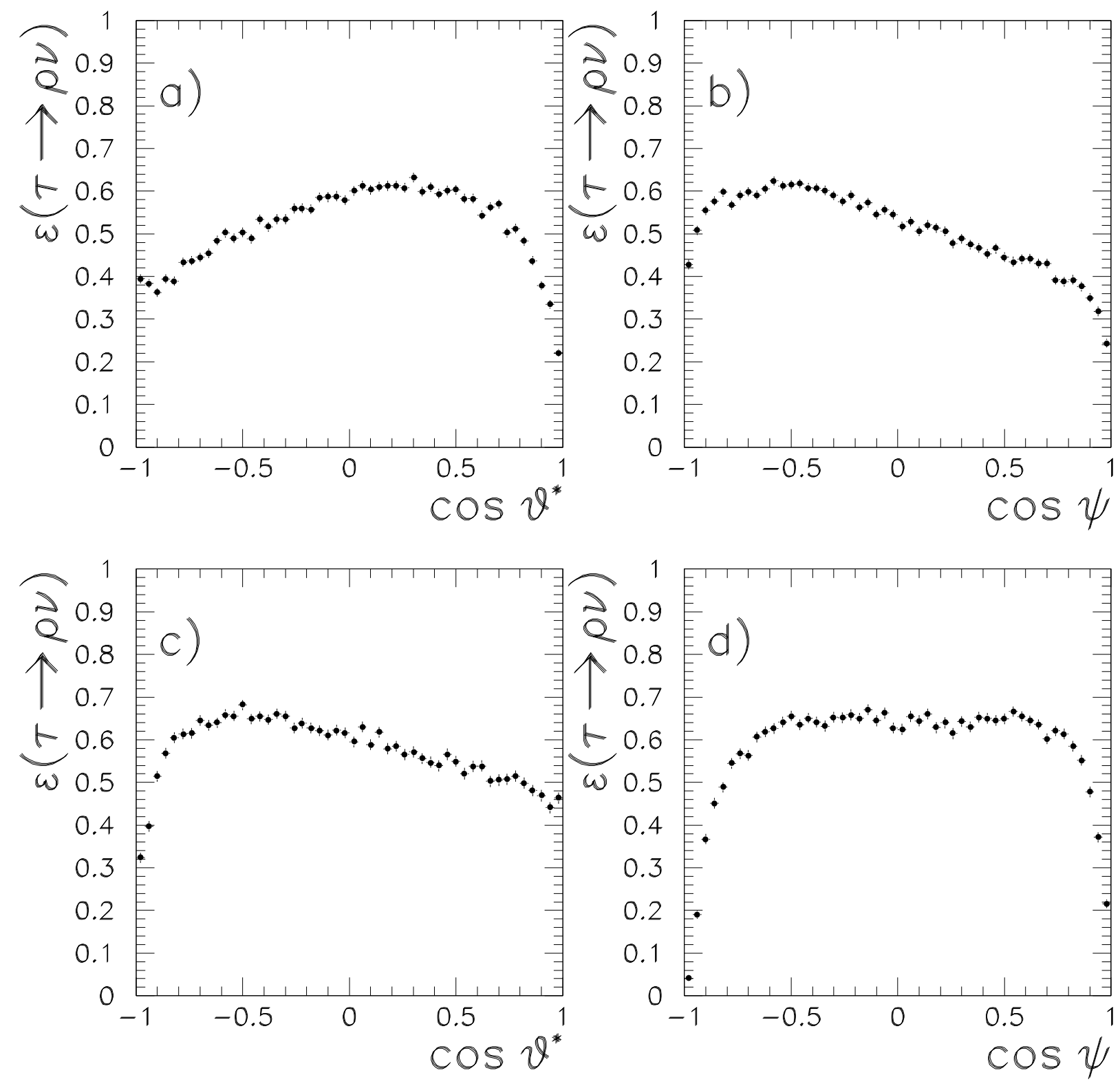

Figure 2: Global efficiencies as a function of (a) $\cos \theta^{*}$ and $(\mathrm{b}) \cos \psi$ for $\tau \rightarrow \rho \nu_{\tau}$ decays for selection I. The analogous plots for selection II are shown in $(c)$ and $(d)$. The efficiencies include the $\tau$-pair selection efficiency and are evaluated after making the fiducial cut: $|\cos \theta|<0.68$. 
OPAL
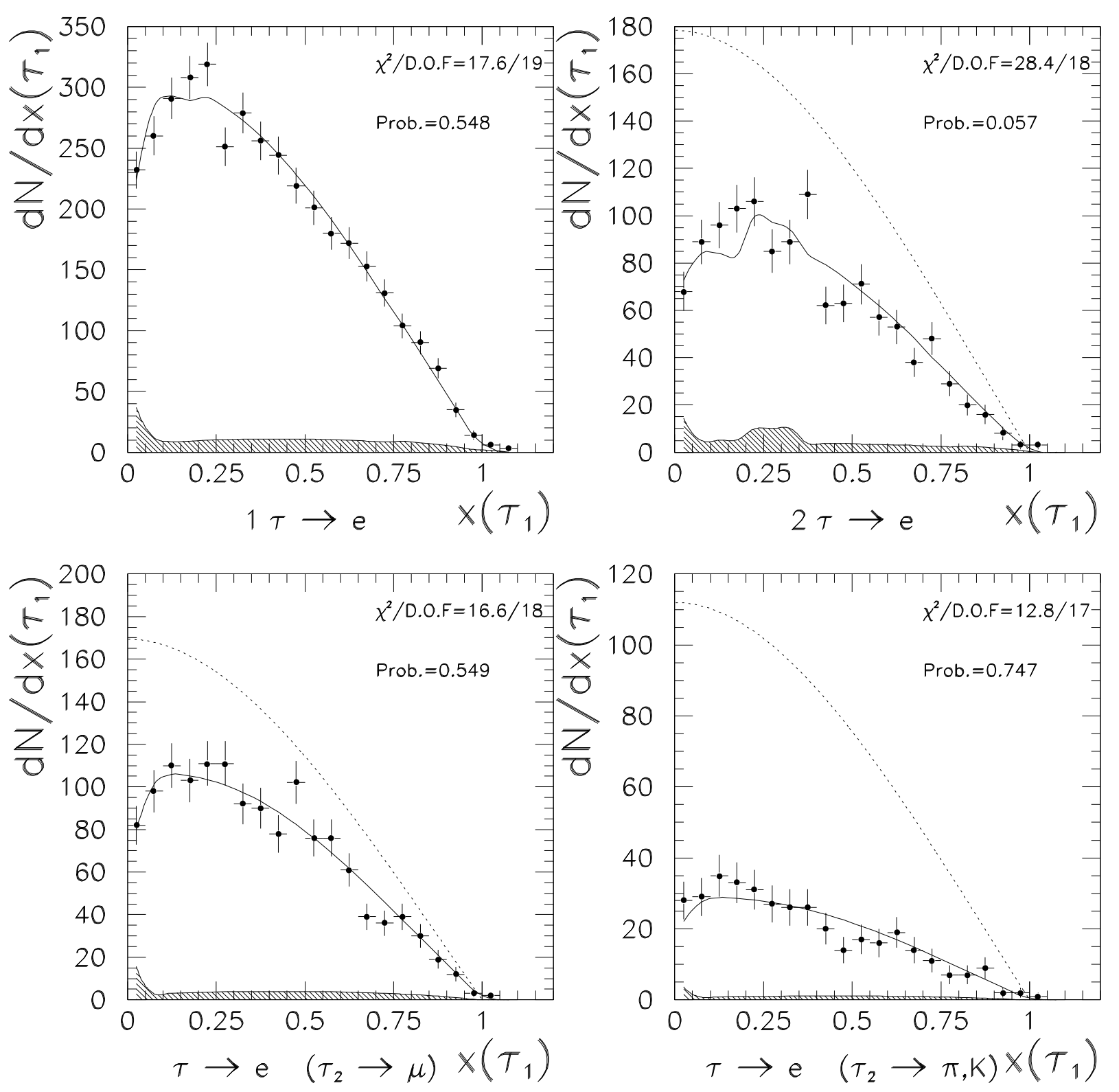

Figure 3: $x$ distributions for the $\tau \rightarrow \mathrm{e}_{e} \nu_{\tau}$ data (points with error bars) for various decays on the opposite side, compared with the theoretical predictions (solid lines) with all corrections included, as calculated with the $\left\langle P_{\tau}\right\rangle$ value obtained from the global maximum likelihood fit. The hatched area denotes the background part of the spectrum and, for the cases where both $\tau$ decays are identified, the dotted lines show the uncorrected theoretical curves. 

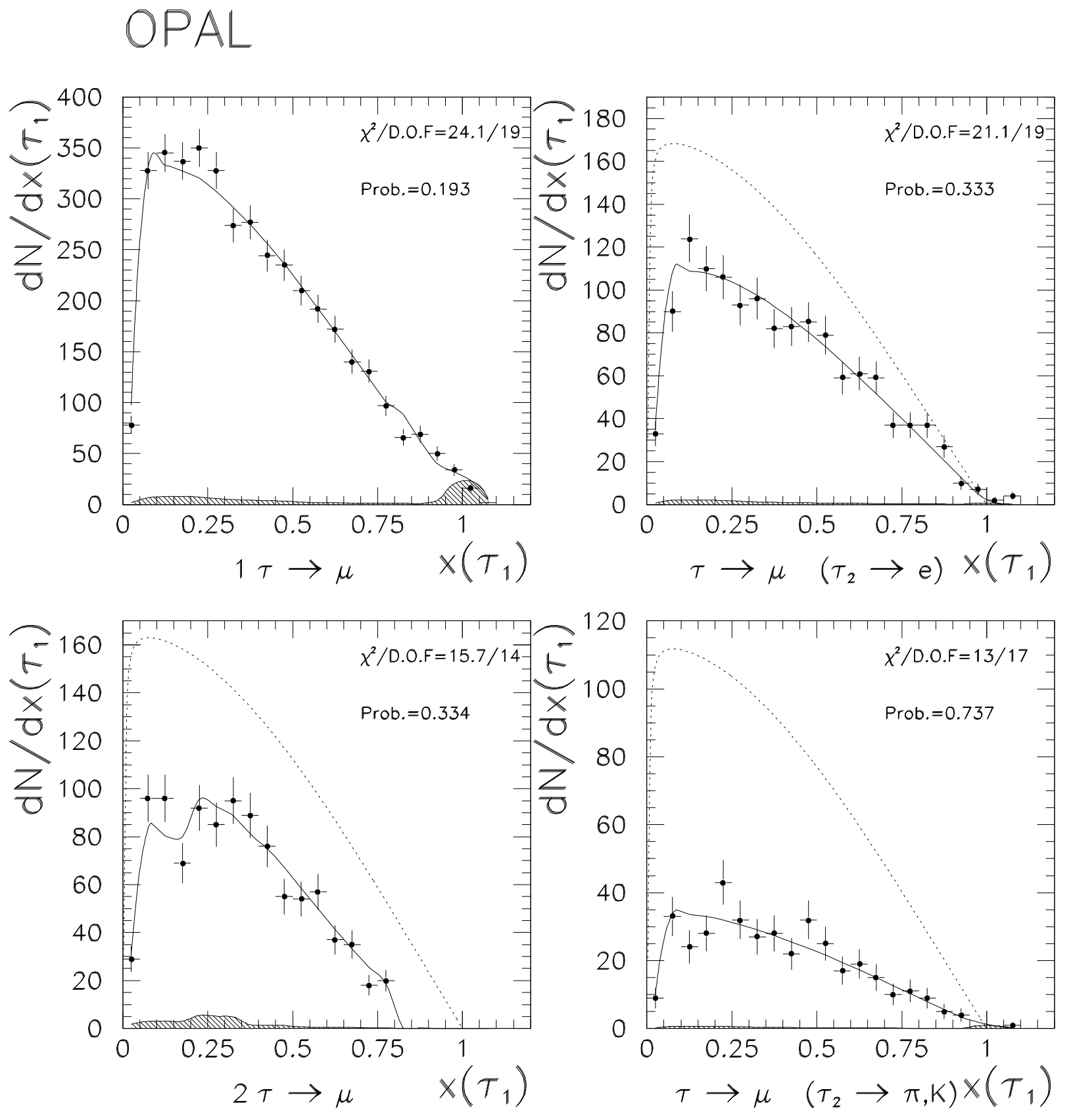

Figure 4: $x$ distributions for the $\tau \rightarrow \mu \bar{\nu}_{\mu} \nu_{\tau}$ data (points with error bars) for various decays on the opposite side, compared with the theoretical predictions (solid lines) with all corrections included, as calculated with the $\left\langle P_{\tau}\right\rangle$ value obtained from the global maximum likelihood fit. The hatched area denotes the background part of the spectrum and, for the cases where both $\tau$ decays are identified, the dotted lines show the uncorrected theoretical curves. 

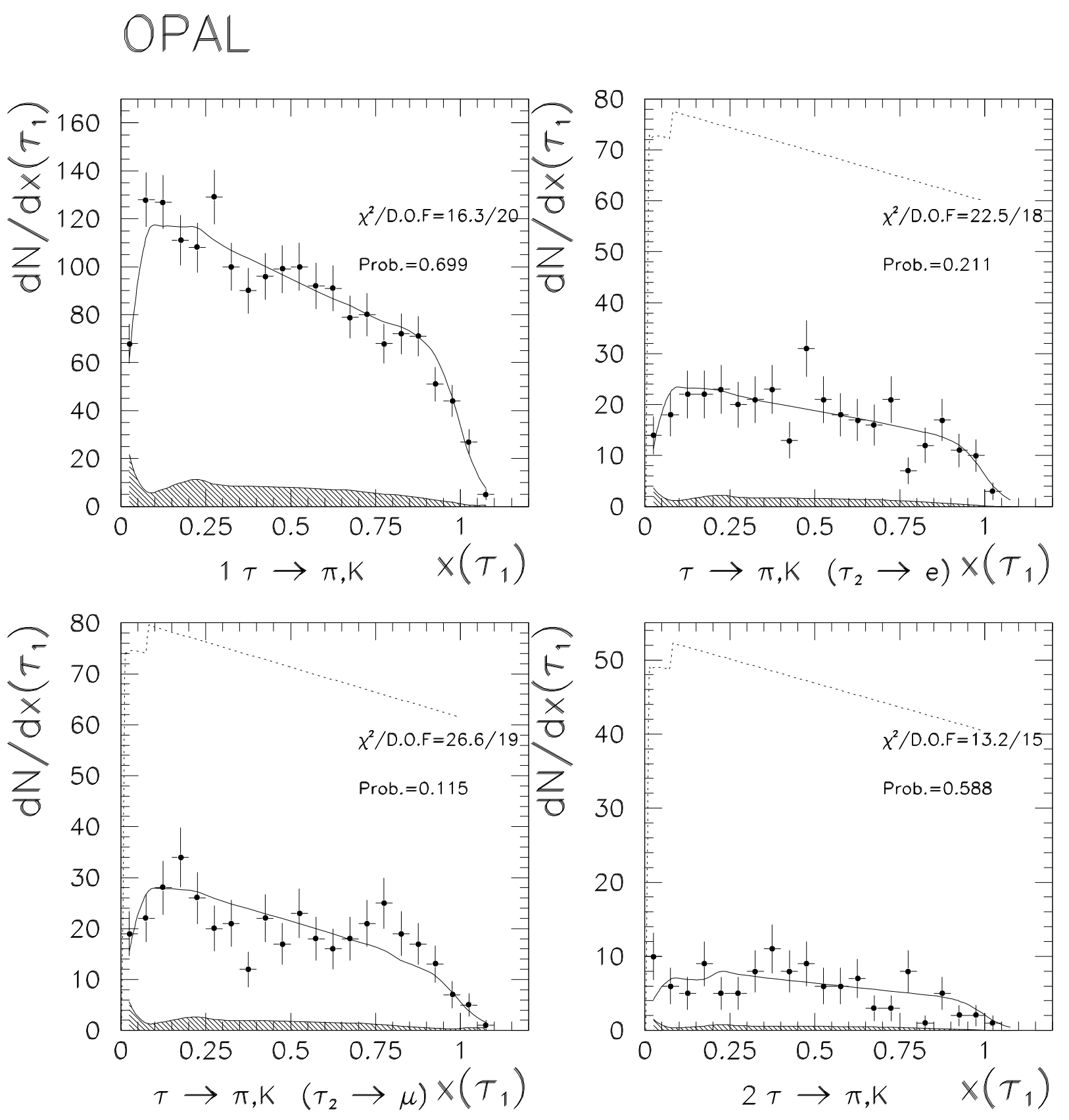

Figure 5: $x$ distributions for the $\tau \rightarrow \pi(\mathrm{K}) \nu_{\tau}$ data (points with error bars) for various decays on the opposite side, compared with the theoretical predictions (solid lines) with all corrections included, as calculated with the $\left\langle P_{\tau}\right\rangle$ value obtained from the global maximum likelihood fit. The hatched area represents the background part of the spectrum and, for the cases where both $\tau$ decays are identified, the dotted lines show the uncorrected theoretical curves. 

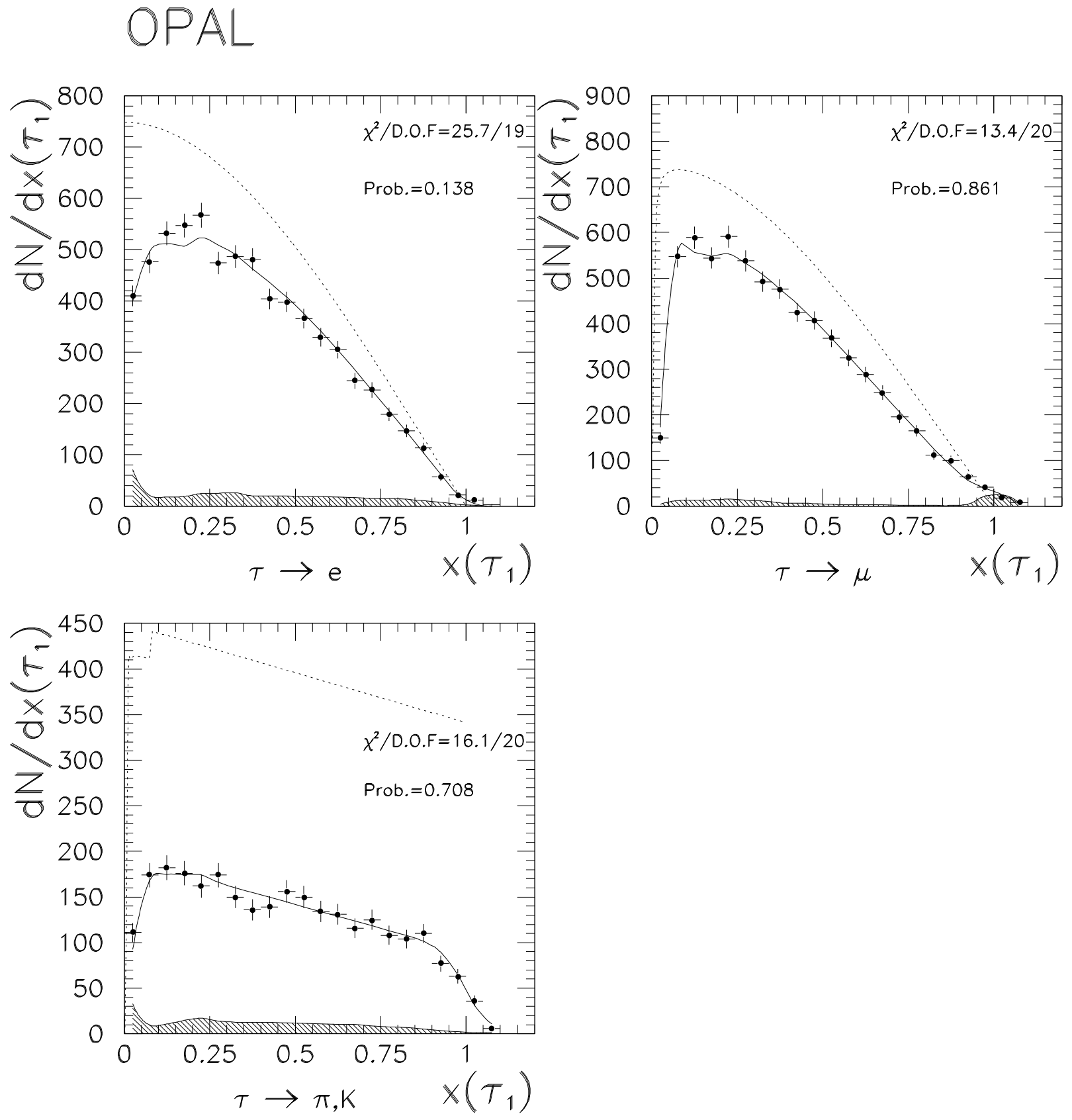

Figure 6: $x$ distributions for the $\tau \rightarrow \mathrm{e} \bar{\nu}_{e} \nu_{\tau}, \tau \rightarrow \mu \bar{\nu}_{\mu} \nu_{\tau}$ and $\tau \rightarrow \pi(\mathrm{K}) \nu_{\tau}$ decay channels, summing over all decays on the other side and compared with the theoretical predictions (solid lines) with all corrections included, as calculated with the $\left\langle P_{\tau}\right\rangle$ value obtained from global fit. The points with error bars represent the data, the hatched area represents the background part of the spectrum and the dotted lines show the uncorrected theoretical curves. 


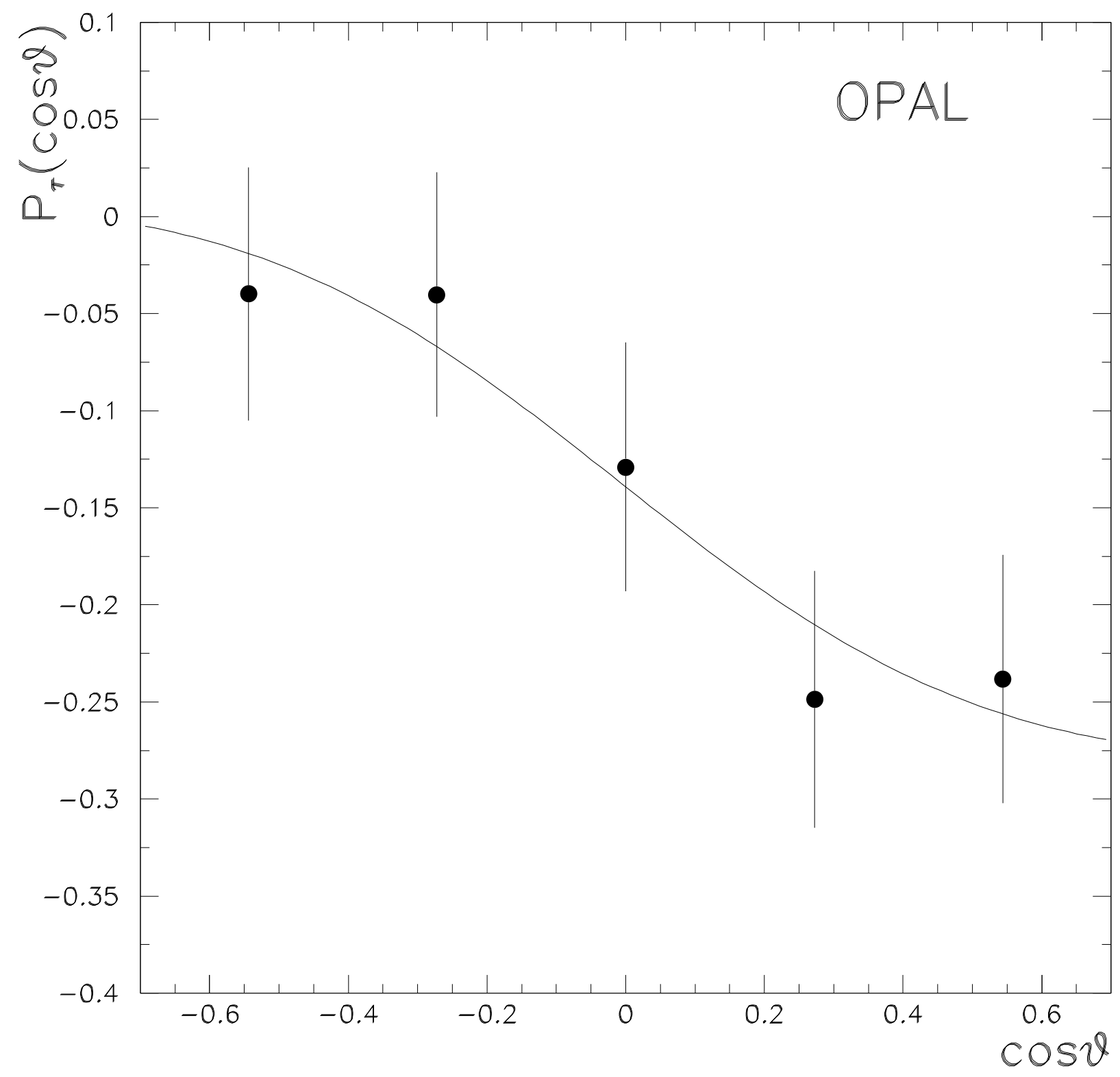

Figure 7: Tau polarization as function of $\cos \theta$ for the $\tau \rightarrow \mathrm{e} \bar{\nu}_{e} \nu_{\tau}, \tau \rightarrow \mu \bar{\nu}_{\mu} \nu_{\tau}$ and $\tau \rightarrow \pi(\mathrm{K}) \nu_{\tau}$ decays. The data points represent the $\left\langle P_{\tau}\right\rangle_{\theta}$ values calculated using a modified maximum likelihood fit in a special analysis used to demonstrate the fit quality and to cross-check the global fit, as described in the text. The solid line is the result of the global maximum likelihood fit. Note that the error bars represent statistical uncertainties only. 
OPAL
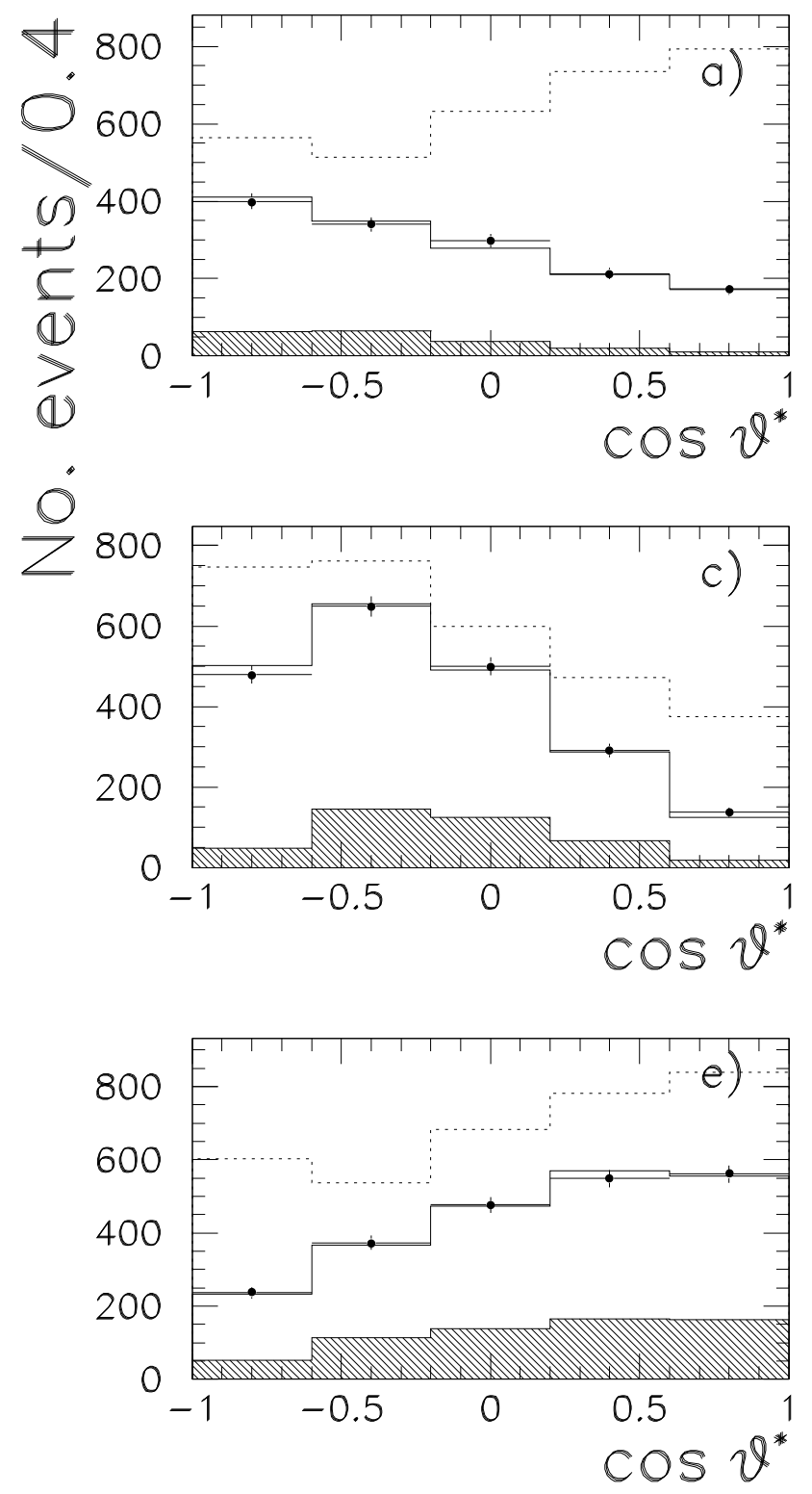
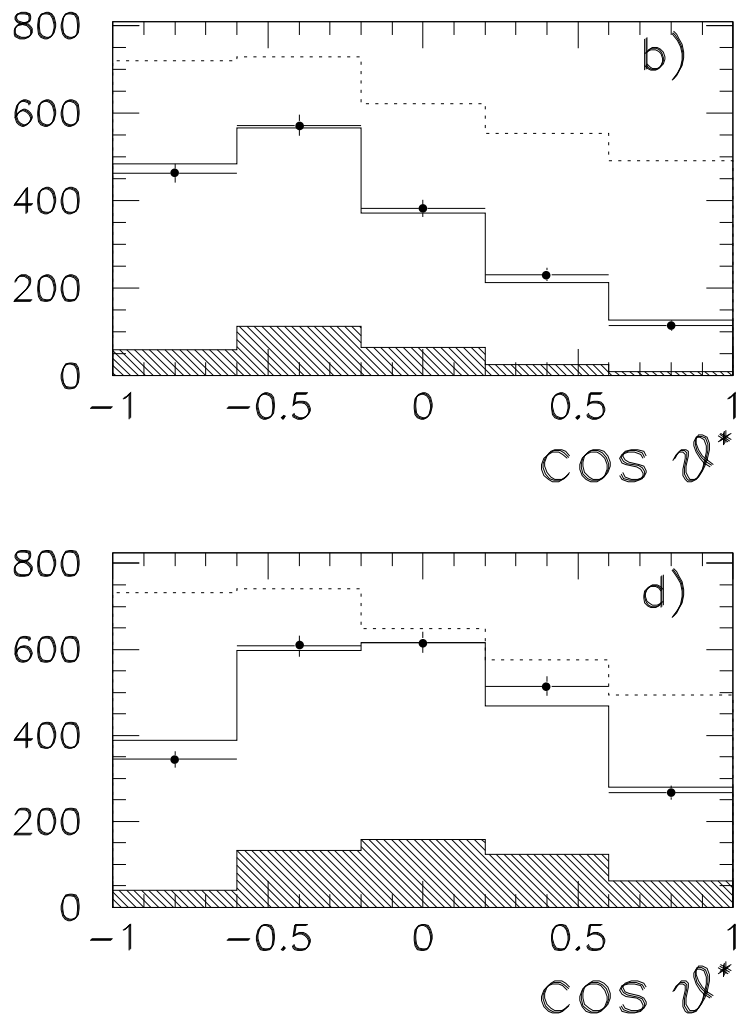

Figure 8: Distributions in $\cos \theta^{*}$ in different $\cos \psi$ regions for the $\tau \rightarrow \rho \nu_{\tau}$ selection I data. The open histograms represent the fitted theoretical curve after applying all corrections. The hatched area represents the background component. The dotted histograms represent the theoretical curve before applying any corrections. The distribution in $\cos \theta^{*}$ for $\cos \psi$ in the range a) $[-1.0,-0.6]$, b) $[-0.6,-0.2]$, c) $[-0.2,+0.2]$, d) $[+0.2,+0.6]$ and e) $[+0.6,+1.0]$ are presented. Note that all bins in $\cos \theta$ have been combined to make these plots. 

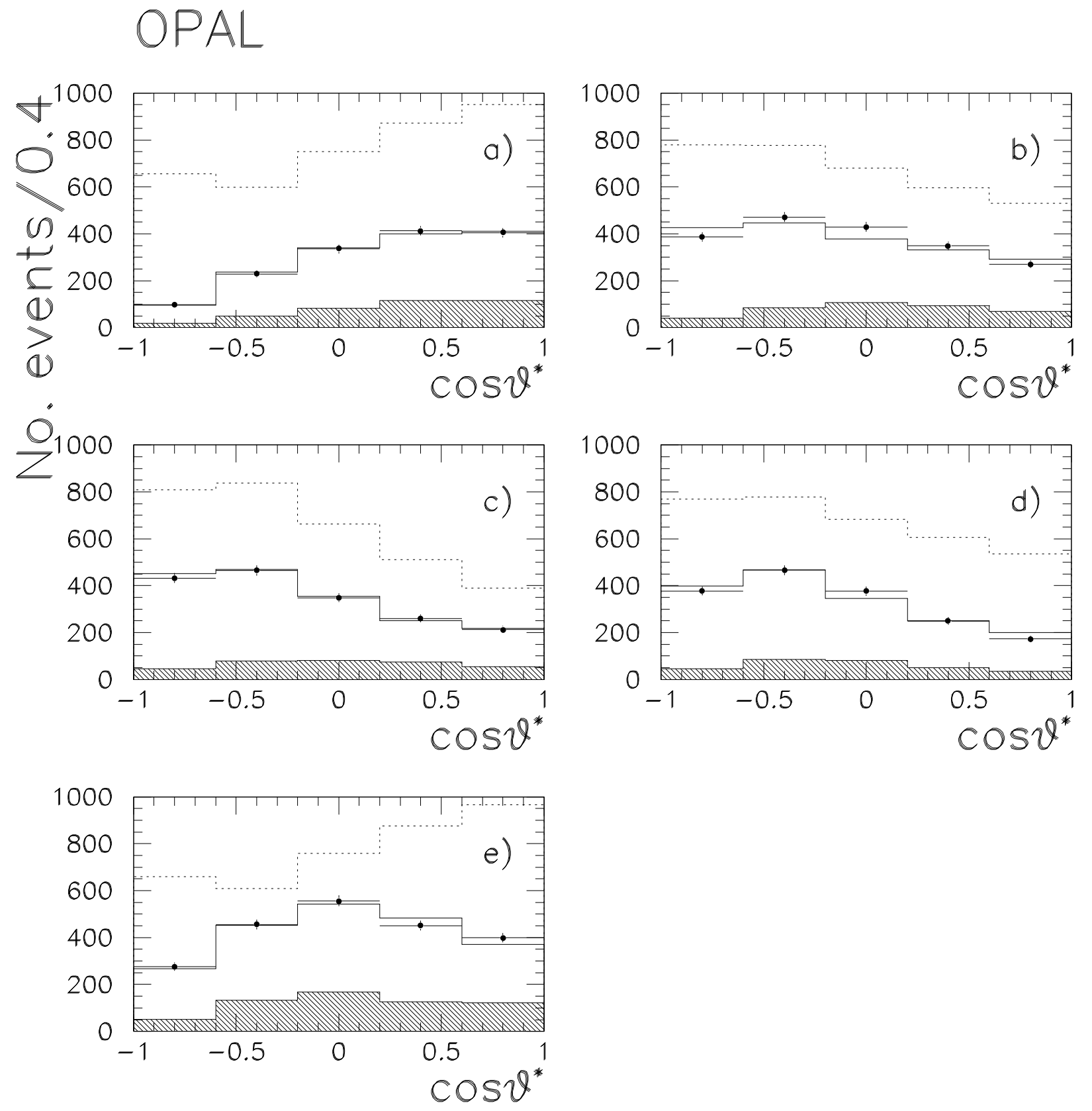

Figure 9: Distributions in $\cos \theta^{*}$ in different $\cos \psi$ regions for the $\tau \rightarrow \rho \nu_{\tau}$ selection II data. The open histograms represent the fitted theoretical curve after applying all corrections. The hatched area represents the background component. The dotted histograms represent the theoretical curve before applying any corrections. The distribution in $\cos \theta^{*}$ for $\cos \psi$ in the range a) $[-1.0,-0.6]$, b) $[-0.6,-0.2]$, c) $[-0.2,+0.2], \mathrm{d})[+0.2,+0.6]$ and $\mathrm{e})[+0.6,+1.0]$ are presented. Note that all bins in $\cos \theta$ have been combined to make these plots. 

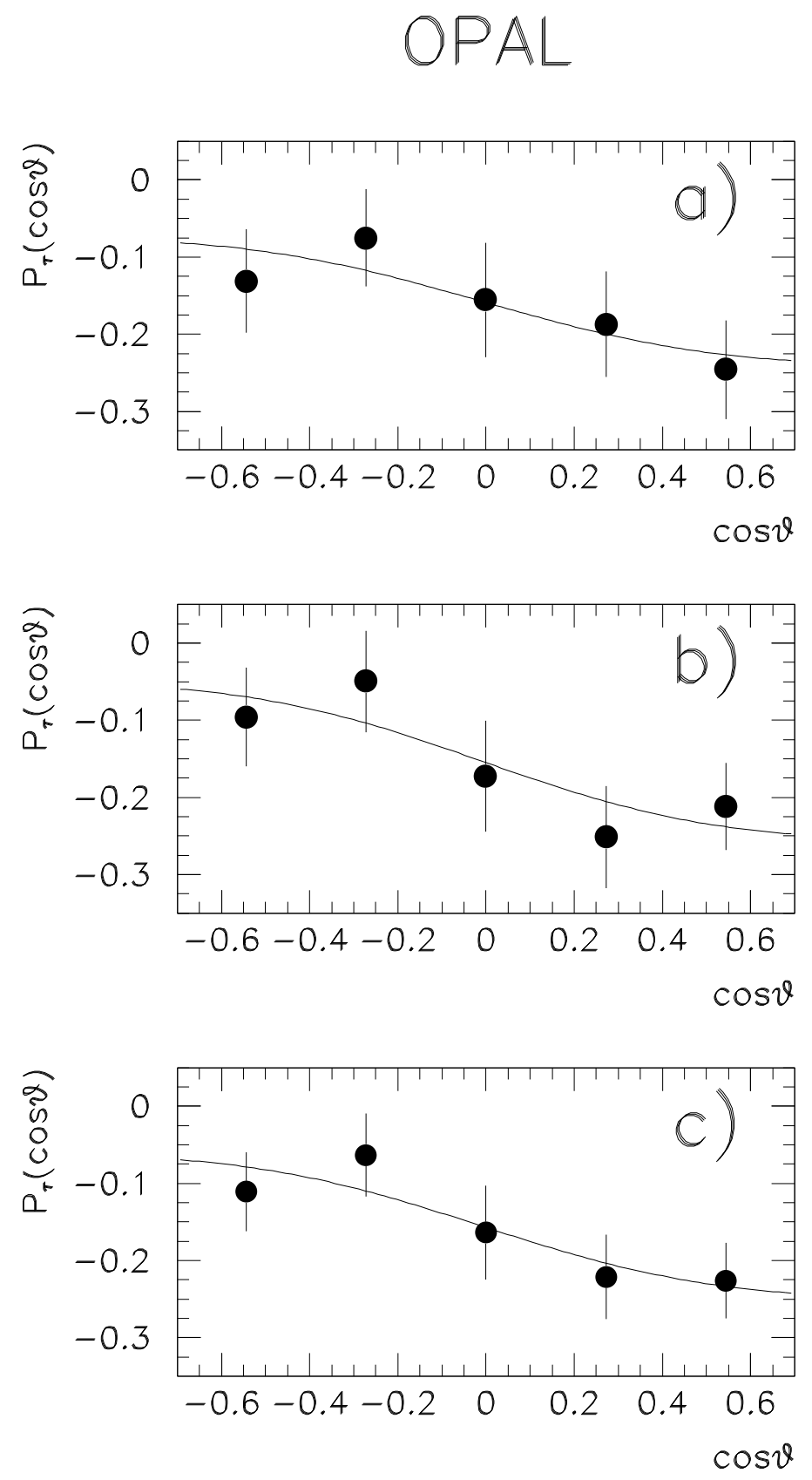

Figure 10: Tau polarization results from the $\tau \rightarrow \rho \nu_{\tau}$ analyses as a function of $\cos \theta$. The data points represent the $\left\langle P_{\tau}\right\rangle_{\theta}$ values calculated from the analyses of selection I (a) and selection II (b). The solid lines represent the expectations from the separate $\tau \rightarrow \rho \nu_{\tau}$ results. The $\left\langle P_{\tau}\right\rangle_{\theta}$ values calculated from the combined $\tau \rightarrow \rho \nu_{\tau}$ analyses are presented in $(\mathrm{c})$ where the points repesent the data and the solid line represents the expectation from the combined $\tau \rightarrow \rho \nu_{\tau}$ result. 


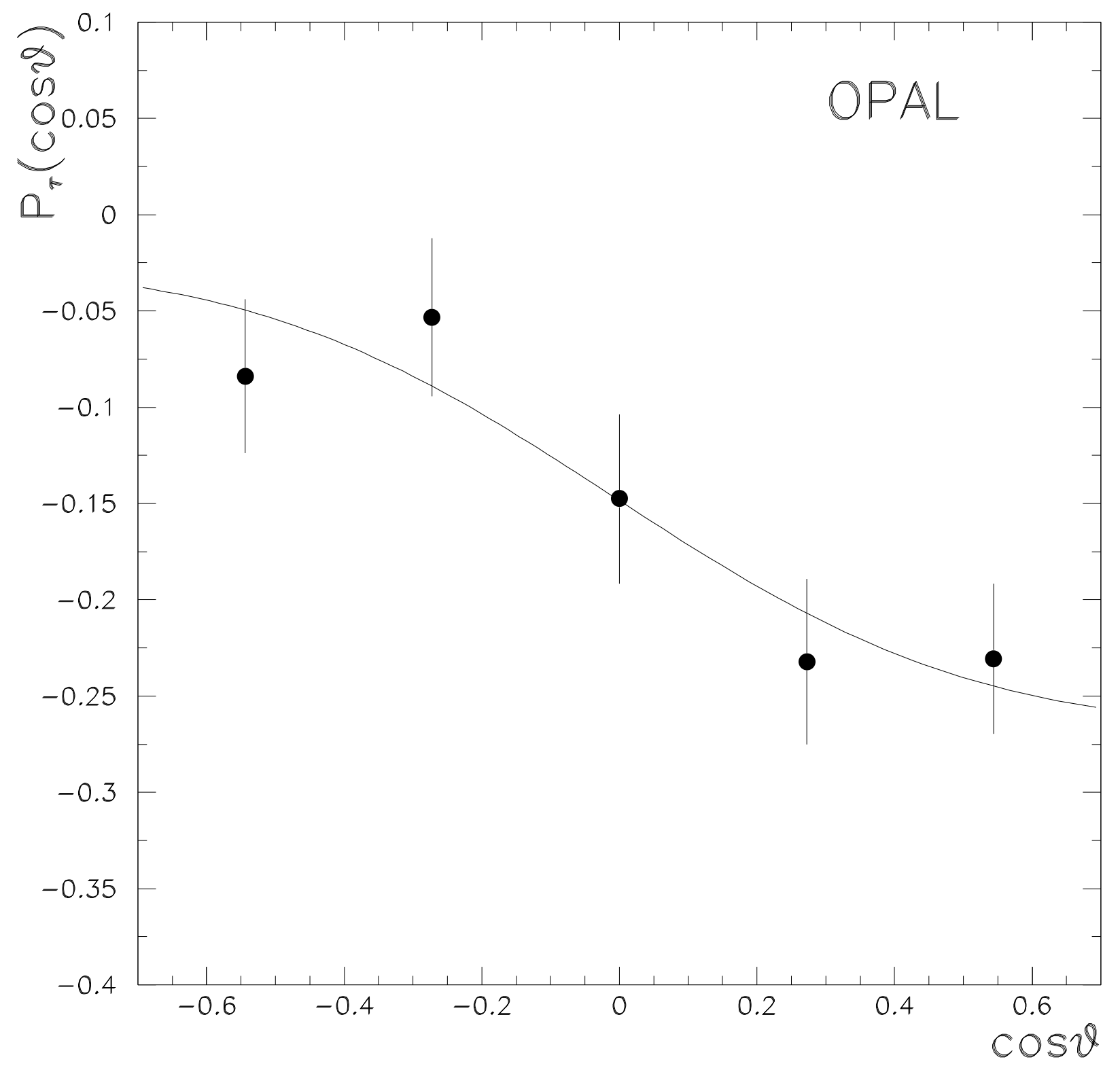

Figure 11: Combined tau polarization results as a function of $\cos \theta$. The data points represent the $\left\langle P_{\tau}\right\rangle_{\theta}$ values calculated as the weighted mean of the global fit and $\tau \rightarrow \rho \nu_{\tau}$ analyses. The solid line represents the expectation from the overall combined result. Note that the error bars represent statistical errors only. 